\title{
Skin-derived neural precursors competitively generate functional myelin in adult demyelinated mice
}

\author{
Sabah Mozafari, ${ }^{1,2,3,4}$ Cecilia Laterza, ${ }^{5}$ Delphine Roussel, ${ }^{1,2,3,4}$ Corinne Bachelin, ${ }^{1,2,3,4}$ Antoine Marteyn, ${ }^{1,2,3,4}$ Cyrille Deboux, ${ }^{1,2,3,4}$ \\ Gianvito Martino, ${ }^{5}$ and Anne Baron-Van Evercooren ${ }^{1,2,3,4}$
}

IINSERM, U1127, F-75013, Paris, France. ²CNRS, UMR 7225, F-75013, Paris, France. ${ }^{3}$ Sorbonne Universités UPMC Univ Paris 06, UM-75, F-75005, Paris, France.

${ }^{4}$ ICM-CH Pitié-Salpêtrière, F-75013, Paris, France. ${ }^{5}$ Institute of Experimental Neurology-DIBIT 2, Division of Neuroscience, San Raffaele Scientific Institute, Milan, Italy.

\begin{abstract}
Induced pluripotent stem cell-derived (iPS-derived) neural precursor cells may represent the ideal autologous cell source for cell-based therapy to promote remyelination and neuroprotection in myelin diseases. So far, the therapeutic potential of reprogrammed cells has been evaluated in neonatal demyelinating models. However, the repair efficacy and safety of these cells has not been well addressed in the demyelinated adult CNS, which has decreased cell plasticity and scarring. Moreover, it is not clear if these induced pluripotent-derived cells have the same reparative capacity as physiologically committed CNS-derived precursors. Here, we performed a side-by-side comparison of CNS-derived and skin-derived neural precursors in culture and following engraftment in murine models of adult spinal cord demyelination. Grafted induced neural precursors exhibited a high capacity for survival, safe integration, migration, and timely differentiation into mature bona fide oligodendrocytes. Moreover, grafted skin-derived neural precursors generated compact myelin around host axons and restored nodes of Ranvier and conduction velocity as efficiently as CNS-derived precursors while outcompeting endogenous cells. Together, these results provide important insights into the biology of reprogrammed cells in adult demyelinating conditions and support use of these cells for regenerative biomedicine of myelin diseases that affect the adult CNS.
\end{abstract}

\section{Introduction}

In CNS myelin disorders, myelin repair prevents axonal loss and leads to functional recovery. Animal models of demyelinating diseases provided overwhelming proofs for the capacity of multipotent neural stem/precursor cells (NPCs) to regenerate and remyelinate CNS axons. Moreover, NPCs provide immunomodulation in EAE, an animal model of multiple sclerosis (MS). These seminal observations suggest that such cells represent a plausible cellular source for cell-based therapy of myelin disorders (1). Although several studies highlighted the impressive therapeutic potential of human fetal NPCs (2-5), the allogeneic nature of the available NPCs has prevented the bench-tobedside translation of NPC-based therapy for these diseases. In search of an accessible, renewable, and nonimmunogenic source of myelin-forming cells, reprogramming strategies were designed to generate rodent or primate induced pluripotent stem cell-derived NPCs (iPS-NPCs) or oligodendrocyte progenitor cells (iPS-OPCs) (6-8). Alternatively, somatic cells were directly reprogrammed into NPCs (iNPCs) (9-13) or OPCs (iOPCs) (14, 15). Few of these studies addressed the capacity of the derived cells to differentiate into oligodendrocytes in vitro or in vivo after engraftment in models of congenital dysmyelination $(8,14,15)$. While, in most cases, the extent to which these cells differentiated into myelin-forming oligodendrocytes was limited, especially for directly reprogrammed cells (iOPCs, iNPCs), multiple

Conflict of interest: The authors have declared that no conflict of interest exists. Submitted: March 24, 2015; Accepted: July 7, 2015.

Reference information: J Clin Invest. 2015;125(9):3642-3656. doi:10.1172/JCI80437. injections of human iPS-OPCs resulted in the entire colonization and myelination of the host dysmyelinated shiverer brain (8). However, in these studies, iPS glial derivatives were transplanted neonatally, taking advantage of the promyelinating cellular and molecular cues, which prevail in the murine brain during the first postnatal weeks of life.

Furthermore, in most of these studies, grafted cells were already committed to OPCs in vitro and likely less competent than NPCs in terms of differentiation plasticity and migration $(16,17)$ - functions that are required to warrant successful remyelination of far-distant lesions of the adult CNS. Finally, whether pluripotent-reprogrammed NPCs behave as genuine CNS cells remains elusive.

Recent data reported that mouse iPS-derived NPCs (miPS-derived NPCs), such as brain-derived NPCs, provide neuroprotection and promotion of endogenous remyelination via leukemia inhibitory factor after intrathecal delivery in a model of immune-mediated demyelination (18). To date, the remyelination potential and safety of iPS-derived NPCs after transplantation into the adult demyelinated white matter - a condition associated with decreased tissue plasticity and trophic support, and encountered in several adulthood demyelinating diseases - were not addressed. Understanding the behavior of iPS-NPCs and namely their time course of differentiation and myelination when facing the adult demyelinating CNS remain key issues for successful translation of iPS-based therapies to the clinic. Here, we took advantage of the demyelinating agent lysolecithin to specifically target myelin in the adult spinal cord. We then used Sox $2^{\text {ggeo/ } \beta \text { geo }}$ mice (herein referred to as Sox $2 \beta$ Geo mice) as a means 

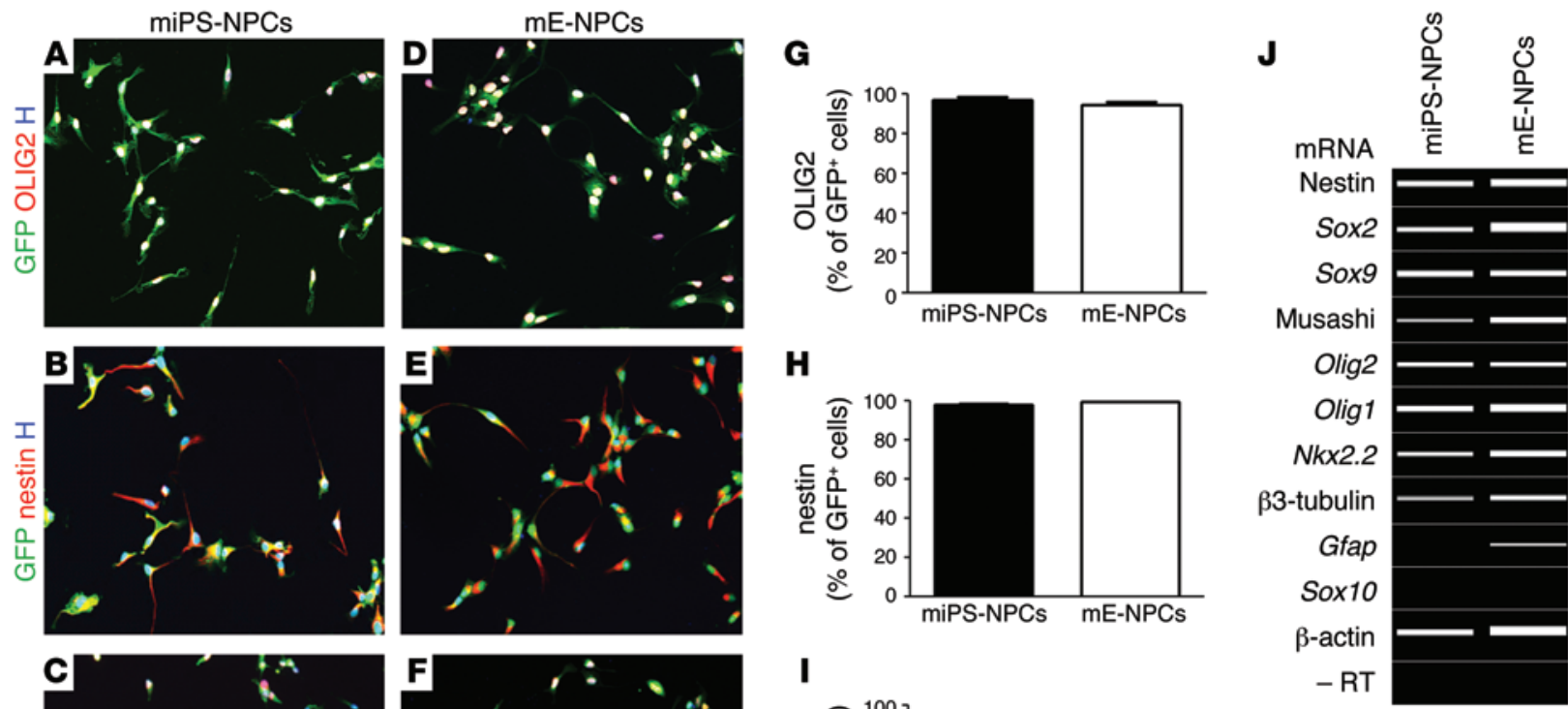

\section{H}
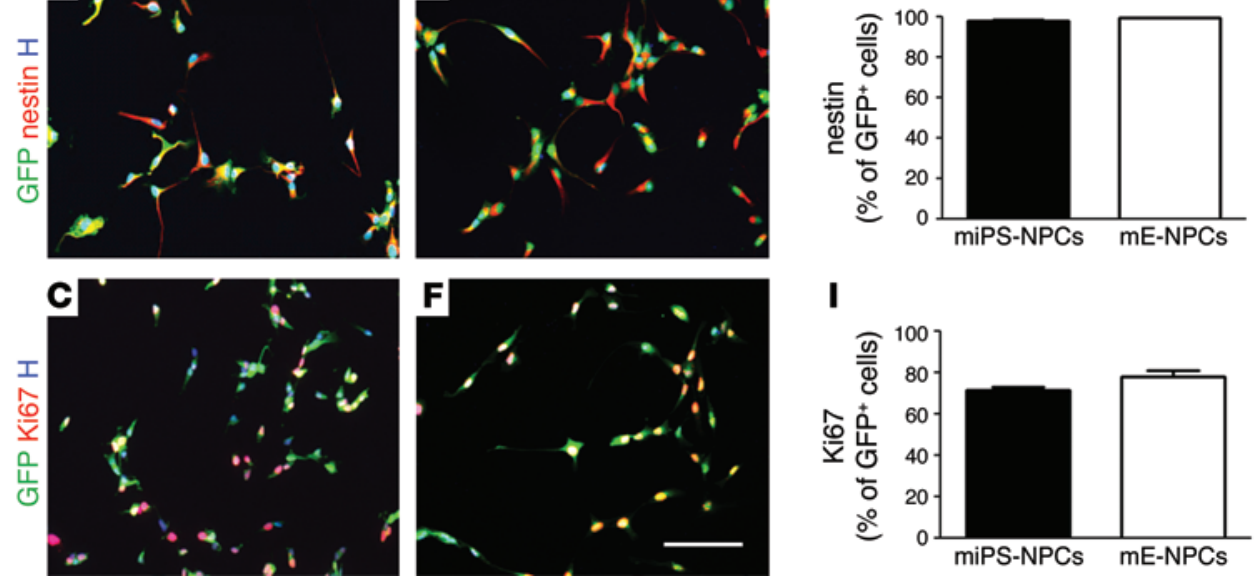

|

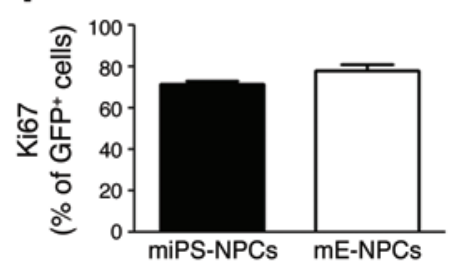

Figure 1. miPS-NPCs have phenotypic characteristics of immature neural progenitor cells in vitro. (A-F) Immunocytochemical characterization of miPS-NPCs (A-C) and mE-NPCs (D-F) illustrating comparable expression of immature markers OLIG2, nestin, and proliferation marker Ki67. (G-I) No significant difference was observed following quantification of theses markers. Student's $t$ test was used for the statistical analysis of these experiments. (J) Semi-quantitative RT-PCR analysis, visualized using a Caliper electrophoresis, confirmed that miPS-NPCs and mE-NPCs expressed mainly transcripts associated with immature cells. Cfap mRNA expression was absent in miPS-NPCs. A-J were performed in 3 independent experiments. H, Hoechst dye. Scale bars: $100 \mu \mathrm{m}$.

to efficiently and safely select miPS-NPCs and compared them with mouse embryonic CNS-derived NPCs (mE-NPCs), in vitro and in vivo. We report that, upon transplantation in the adult demyelinated spinal cord, skin-derived NPCs integrate structurally and functionally into the adult CNS parenchyma and in a manner indistinguishable from genuine CNS-derived NPCs. The impressive therapeutic benefit of miPS-NPCs on CNS remyelination and recovery of axonal dysfunction suggest promising perspectives for personalized myelin-replacement therapy using the patient's own cells in myelin disorders of the adult CNS.

\section{Results}

miPS-NPCs express immature neural cell markers of CNS-derived cells at the transcript and protein levels. NPCs were derived from miPS cells obtained from Sox $2 \beta G e o$ mice and previously characterized in vitro for their ability to self-renew and give rise to neurons, astrocytes, and oligodendrocytes when exposed to differentiation conditions (18). To further study miPS-NPCs in vitro, cells were stained with different markers of immature neural cells (nestin, Ki67, and OLIG2) and mature neural cells (GFAP, PDGFR $\alpha, \mathrm{O} 4, \mathrm{MAP} 2$, and $\beta 3$-tubulin), and they were compared with brain-derived mE-NPCs. The percentages of $\mathrm{GFP}^{+}$cells expressing these markers in 3 independent experiments showed that miPS-NPCs expressed essentially OLIG2 (96.97\% $\pm 1.6 \%)$, nestin $(98.19 \% \pm 0.52 \%)$, and Ki67 $(71.41 \% \pm 1.8 \%$ ) (Figure $1, \mathrm{~A}-\mathrm{C})$. These values were similar to those of mE-NPCs with $94.52 \pm 1.4,99.52 \pm 0.10$, and 78.18 \pm 3.1, respectively (Figure $1, \mathrm{D}-\mathrm{I}$ ). Furthermore, most miPSNPCs expressed vimentin, with few cells expressing $\beta 3$-tubulin and no cells expressing the more mature markers of glial cells (GFAP, PDGFR $\alpha$, and O4) or neurons (MAP2) (Supplemental Figure 1; supplemental material available online with this article; doi:10.1172/JCI80437DS1). Transcript expression analysis confirmed the immunocytochemical characterization. miPS-NPCs expressed transcripts for nestin, Sox2, Sox9, musashi, Olig2, Olig1, and Nkx2.2, but not for Gfap and Sox10 (Figure 1J). Similar data were recorded for mE-NPCs, except for GFAP, which they expressed at the antigen (Supplemental Figure 1L) and transcriptional levels (Figure 1J).

miPS-NPCs survive and integrate successfully following transplantation into the demyelinated adult CNS. To investigate the behavior of miPS-NPCs in the context of demyelination, LPC-induced demyelination was targeted to the dorsal funiculus of adult immunodeficient nude (with normal myelination) or Mbphishi Rag2-/- (with congenital dysmyelination, herein referred to as Shi/Shi Rag2-/-) mice, and cells were transplanted into the lesion site 48 hours after demyelination using previously described methods (Figure 2A; ref. 2). Lesions were similar in size at 1 week postgraft (wpg), $42.19 \% \pm$ $2.394 \%, 52.38 \% \pm 2.902 \%$, and $51.31 \% \pm 5.756 \%$ of the total dorsal funiculus area in nongrafted (medium-treated), miPS-NPCs, and mE-NPC-grafted animals, respectively (Figure 2B). Nude animals were sacrificed 1, 2, and $6 \mathrm{wpg}$ to follow grafted cell dynamics in terms of cell proliferation, specification, and maturation, while Shi/Shi Rag $2^{-/-}$mice were analyzed mainly to follow donor-derived 
A

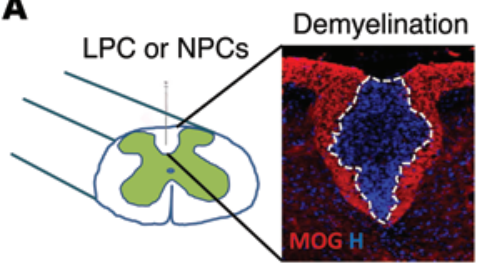

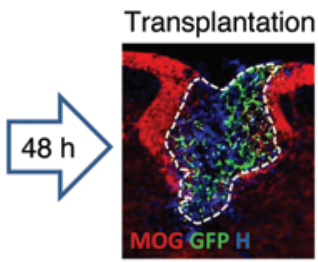

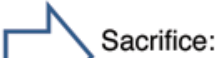

$1,2,6$, and $10 \mathrm{wpg}$
B

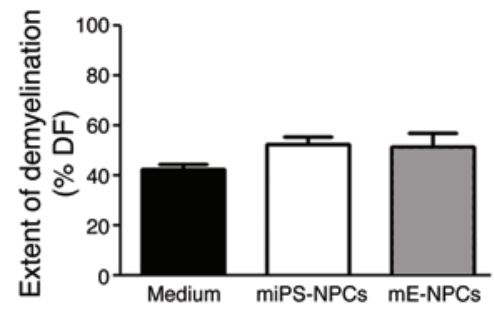

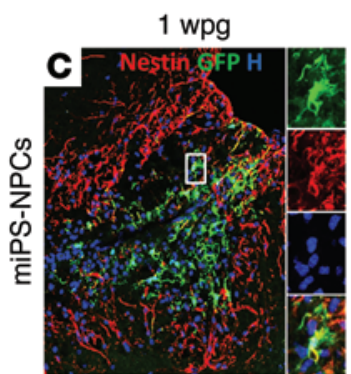
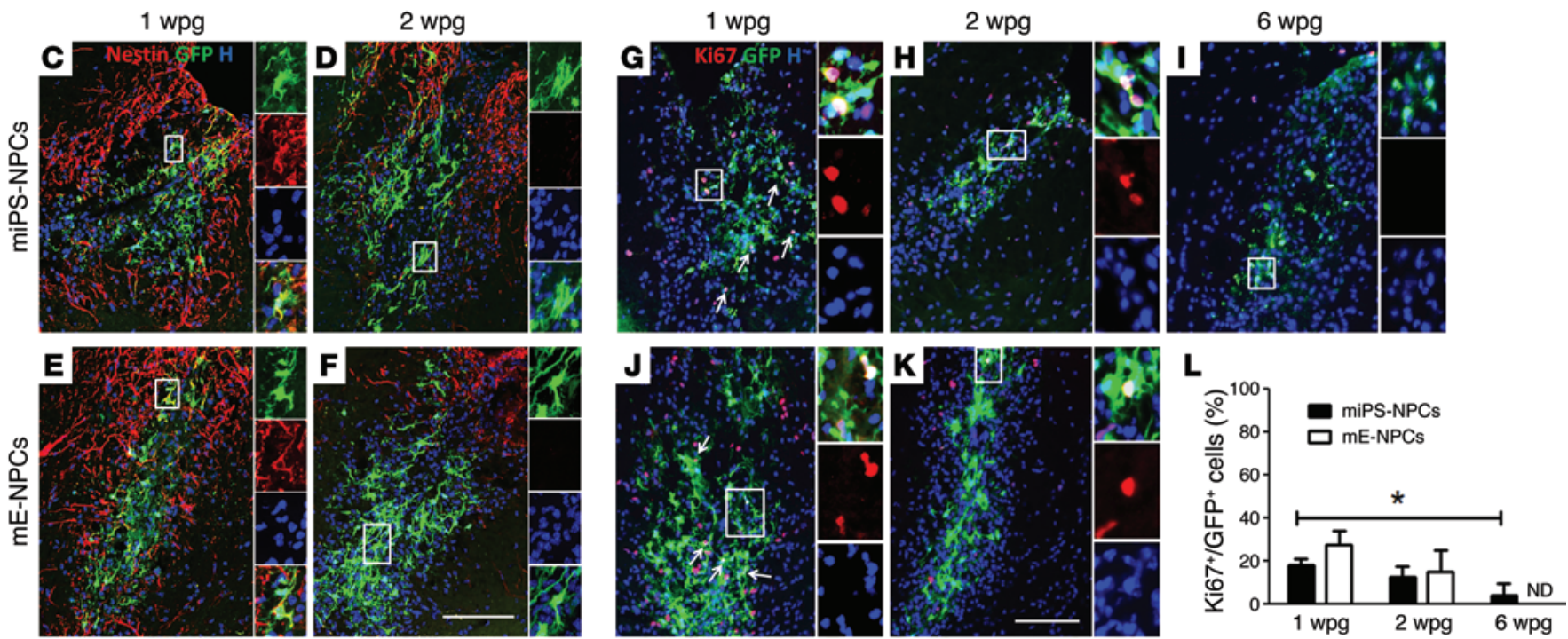

Figure 2. miPS-NPCs decrease expression of immature markers immediately following transplantation in demyelination context. (A) Experimental outline: demyelination (revealed by depletion of MOC staining) was induced by injection of LPC in the dorsal funiculus of the adult nude or Shi/Shi Rag2 ${ }^{-1-}$ mice. miPS-NPCs or mE-NPCs were transplanted in the lesion site 48 hours after demyelination. Animals were sacrificed at 1, 2, 6, and 10 wpg. (B) Quantification of extent of demyelination on coronal section (based on MOC depletion) at 1 wpg showed $40 \%-50 \%$ demyelination in dorsal funiculus (DF) with no significant difference between different groups of medium-treated or grafted mice. (C and D) In the lesion, only few miPS-NPCs expressed the immature marker nestin at 1 wpg and lost this expression beyond 2 wpg. (E and $\mathbf{F})$ mE-NPCs showed similar pattern of nestin expression ( $n=3-4$ mice per time point and per cell type). (G-L) miPS-NPCs significantly decreased expression of Ki67 following 1 wpg $(P=0.026)$ vs. 6 wpg (G-I). No significant difference was observed between the proliferation rates of mE-NPCs (J and $\mathbf{K}$ ) and miPS-NPCs following engraftment (L). One-way analysis of variance plus Tukey's multiple comparison tests and Student's $t$ test were used for the statistical analysis of these experiments ( $n=3-4$ mice per group). ${ }^{*} P<0.05$. H, Hoechst dye; n.d., not done. Scale bars: $100 \mu \mathrm{m}$.

differentiation or myelination at later time points (6 and $10 \mathrm{wpg})$. Grafted cell survival, distribution, and integration were analyzed in both strains and for both cell types throughout the experiments.

$\mathrm{GFP}^{+}$miPS-derived cells were found in all transplanted animals $(n=35)$ at all time points, indicating that cells survived in the host environment up to $10 \mathrm{wpg}$. The density of the grafted $\mathrm{GFP}^{+}$ cells was similar for both cell types at $1 \mathrm{wpg}(210.5 \% \pm 21.63 \%$ and $262.8 \% \pm 33.29 \%$ per $\mathrm{mm}^{2}$, respectively, for miPS-NPCs and mE-NPCs) and $2 \mathrm{wpg}(316.1 \% \pm 27.22 \%$ and $407.4 \% \pm 37.25 \%$ per $\mathrm{mm}^{2}$ for miPS-NPCs and mE-NPCs, respectively), confirming the reproducibility of the lesion/graft paradigm $(n=3-4$ per cell type and time point). Survival of the grafted cells was further confirmed by the absence of caspase 3 expression, a marker of apoptotic activity (Supplemental Figure 2, A and D). Moreover, as observed in vitro (18), miPS-NPCs, similar to mE-NPCs, did not express the pluripotency transcription factor OCT4, used for cell reprogramming (Supplemental Figure 2, B and E), nor NANOG, a marker for both cell pluripotency and tumor progression, up to 10 wpg (Supplemental Figure 2, C and F).

In nude mice, the number of miPS-NPCs expressing the proliferation marker Ki67 was timely downregulated from 1 wpg
$(18.31 \% \pm 1.51 \%)$ and $2 \mathrm{wpg}(12.42 \% \pm 3.11 \%)$ to $6 \mathrm{wpg}(4.32 \% \pm$ $2.95 \%$ ) (Figure 2, G-I, and L). While no significant reduction in proliferation rate was found between 1 and $2 \mathrm{wpg}$, proliferation was significantly decreased at 6 wpg compared with $1 \mathrm{wpg}$ (Figure 2L). Proliferation rates for grafted mE-NPCs at 1 and 2 wpg were respectively $27.36 \% \pm 3.75 \%$ and $15.13 \% \pm 5.62 \%$, with no significant differences with those of miPS-derived cells at the same time points (Figure 2, J-L). miPS-NPCs timely ceased proliferation not only in nude mice, but also in Shi/Shi Rag2 ${ }^{--}$mice, with only $2.77 \% \pm 0.20 \%$ of $\mathrm{GFP}^{+}$cells proliferating at $6 \mathrm{wpg}$ compared with $8.052 \% \pm 1.81 \%$ for mE-NPCs (Supplemental Figure 3, A-C). There was no significant difference between the mitotic behav-

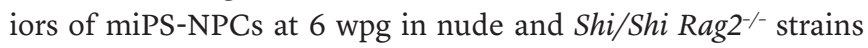
(Supplemental Figure 3D). Consistent with downregulation of proliferation, aberrant cell aggregation or tumors were never observed throughout the experimental time up to $10 \mathrm{wpg}(n=40)$.

miPS-NPCs progressively differentiate into mature oligodendrocytes in adult demyelinating condition. To investigate miPSNPC differentiation potential into oligodendroglial cells, tissues from transplanted animals were stained for the immature marker nestin and markers of different stages of the oligodendroglial 

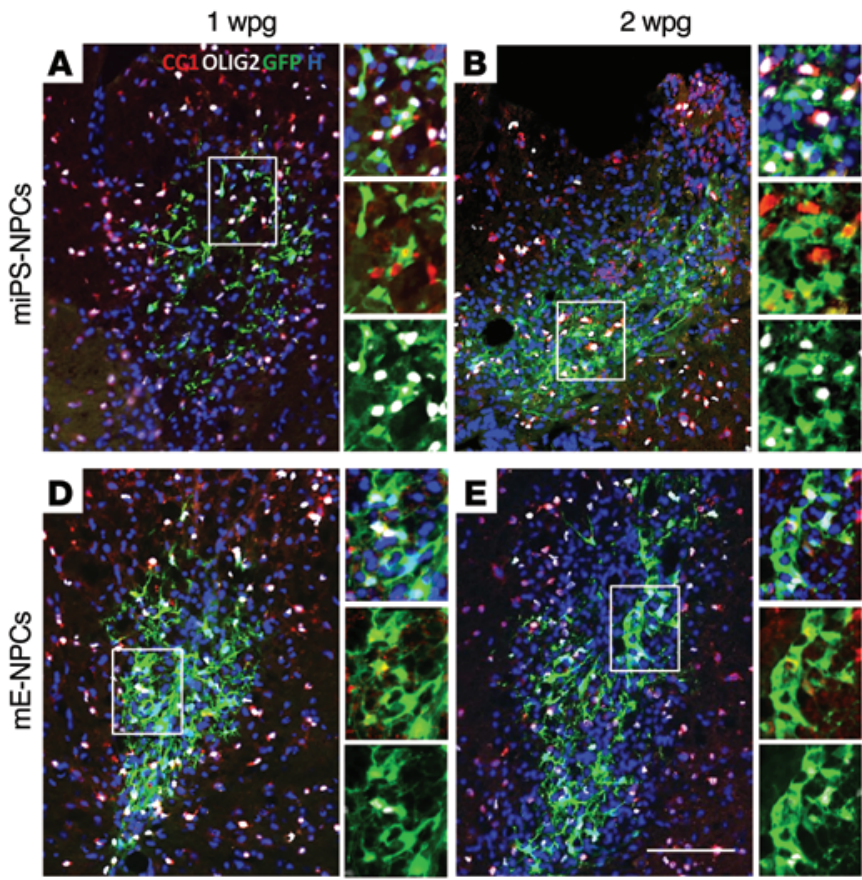

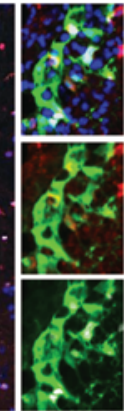

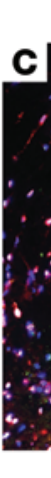

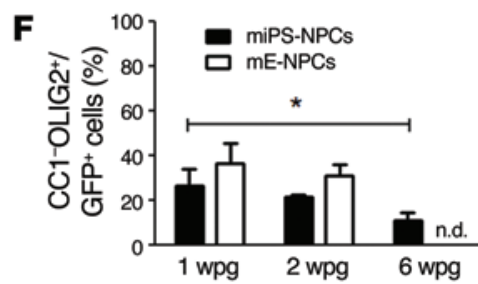

G

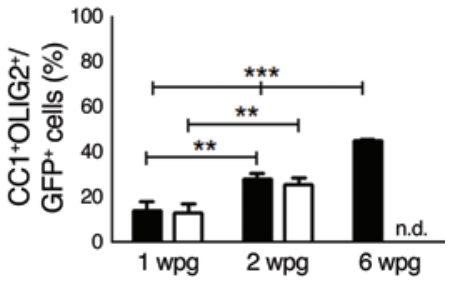

Figure 3. miPS-NPCs differentiate progressively into mature oligodendrocytes in response to demyelination in nude mice. (A-E) Combined immunodetection of OLIG2 (white) and CC1 (red) with GFP (green) for miPS-NPCs (A-C) and mE-NPCs (D and $\mathbf{E}$ ) at 1 ( $\mathbf{A}$ and $\mathbf{D}), 2$ (B and E), and 6 (C) wpg in myelin WT mice. (F and $\mathbf{G})$ Quantification of $\mathrm{CC1}^{-} / \mathrm{OLIC2}^{+}(\mathbf{F})$ and $\mathrm{CC1}^{+} / \mathrm{OLIG2}^{+}$ $\mathrm{GFP}^{+}$cells (G). The percentage of immature oligodendrocytes decreased significantly after 2 wpg for both grafted cell types, while the percentage of mature oligodendrocytes increased for both miPS-NPCs and mE-NPCs. One-way analysis of variance plus Tukey's multiple comparison tests and Student's $t$ test were used for the statistical analysis of these experiments ( $n=3-4$ mice per group). ${ }^{*} P<0.05,{ }^{*} P<0.01$, and ${ }^{* *} P<0.001$. H: Hoechst dye; n.d.: not done. Scale bar: $100 \mu \mathrm{m}$. lineage: OLIG2 and SOX10, as general markers of the lineage; PDGFR $\alpha$ and NG2, markers of oligodendrocyte progenitors; CC1, a marker of preoligodendrocytes; and NogoA, MBP, and MOG, markers of mature oligodendrocytes.

While the majority of miPS-derived cells were nestin ${ }^{+}$in vitro, they stopped expressing this immature marker by $1 \mathrm{wpg}$ in nude mice, as was observed for mE-NPCs, suggesting their ongoing specification into mature cells (Figure 2, C-F). Moreover, in the same mice, the percentage of OLIG2 ${ }^{+} \mathrm{GFP}^{+}$miPSNPCs increased over time from $40.49 \% \pm 6.14 \%$ at 1 wpg to $50.01 \% \pm 0.60 \%$ at $2 \mathrm{wpg}$, and $56.15 \% \pm 1.86 \%$ at $6 \mathrm{wpg}$. In fact, miPS-NPCs grafted in nude mice showed typical characteristics of immature oligodendroglial precursor cells (PDGFR $\alpha^{+}, \mathrm{NG}^{+}$, and $\left.\mathrm{OLIG}^{+} / \mathrm{CC1}^{-}\right)$as soon as they encountered host naked axons at 1 and 2 wpg (Figure 3, A-C, and Supplemental Figure 4). While the percentage of OLIG2 $2^{+} / \mathrm{CC1}^{-}$miPS-NPCs consisted of $26.33 \% \pm 4.57 \%$ and $21.69 \% \pm 0.65 \%$ at $1 \mathrm{wpg}$ and $2 \mathrm{wpg}$, respectively, it was significantly decreased at 6 wpg $(11.09 \% \pm 1.97 \%)$ compared with $1 \mathrm{wpg}$ (Figure 3F). The percentages of OLIG2 ${ }^{+} /$ $\mathrm{CC}^{-} \mathrm{mE}-\mathrm{NPCs}$ at early time points were $36.70 \% \pm 4.99 \%$ and $31.08 \% \pm 2.91 \%$ at $1 \mathrm{wpg}$ and $2 \mathrm{wpg}$, respectively, and were not significantly different (Figure 3, D-F).

On the other hand, the percentage of mature $\mathrm{OLIG}^{+} / \mathrm{CCl}^{+}$ oligodendroglial cells derived from miPS-NPCs significantly increased as a function of time from $14.16 \% \pm 2.21 \%$ of $\mathrm{GFP}^{+}$cells at $1 \mathrm{wpg}$ to $28.32 \% \pm 1.26 \%$ at $2 \mathrm{wpg}$ and $45.06 \% \pm 0.42 \%$ at $6 \mathrm{wpg}$ in nude mice (Figure $3, \mathrm{~A}-\mathrm{C}$, and $\mathrm{G}$ ). A similar significant increase was found for mE-NPCs with $13.21 \% \pm 2.22 \%$ and $25.81 \% \pm 1.74 \%$ of $\mathrm{OLIG}^{+} / \mathrm{CC}^{+} \mathrm{GFP}^{+}$cells at 1 and $2 \mathrm{wpg}$ in nude mice (Figure 3, D, E, and G). In Shi/Shi Rag2-/ mice, no significant difference was observed between the percentage of $\mathrm{OLIG}^{+} / \mathrm{CC}^{+} \mathrm{GFP}^{+}$ $\operatorname{miPS}(76.10 \% \pm 2.87 \%)$ and embryonic-derived cells $(61.88 \% \pm$ $4.33 \%) 6$ wpg (Figure 4, A-C). Interestingly, miPS-derived cells differentiated more into OLIG $2^{+} / \mathrm{CC}^{+}$mature cells at $6 \mathrm{wpg}$ in Shi/Shi Rag2 $\%$ mice $(76.10 \% \pm 2.87 \%)$ compared with nude mice $(45.06 \% \pm 0.42 \%)$ (Figure $3 \mathrm{C}$, Figure $4 \mathrm{~A}$, and Supplemental Figure $6 \mathrm{G})$. The presence of a large number of $\mathrm{SOX} \mathrm{O}^{+} / \mathrm{NogoA}^{+} \mathrm{GFP}^{+}$ miPS-derived cells at the lesion site 6 wpg confirmed this observation in Shi/Shi Rag2-- mice (Supplemental Figure 5).

miPS-NPCs are multipotential but generate mainly oligodendrocytes in adult demyelinating conditions. Our data show that, by 6 wpg in Shi/Shi Rag2 ${ }^{-/}$mice, grafted cells differentiated not only into oligodendrocytes, but also into other major neural cell types, indicating their multipotency (Figure 4). While both cell sources gave rise to astrocytes and neurons, the relative proportion of these mature cell types slightly differed between miPSNPC and mE-NPC. miPS-NPC generated lower numbers of $\mathrm{GFAP}^{+}$astrocytes $(10.22 \% \pm 2.12 \%$, Figure $4 \mathrm{D})$ and more $\mathrm{NeuN}^{+}$ neurons $(2.84 \% \pm 0.60 \%$, Figure $4 \mathrm{G})$ than $\mathrm{mE}$-NPCs, which generated more $\mathrm{GFAP}^{+}$cells $(18.07 \% \pm 0.34 \%$ ) (Figure 4 , E and F) and fewer (though not significant) neurons $(1.04 \% \pm 0.57 \%)$ (Figure $4 \mathrm{H})$. However, both cell sources differentiated mainly into oligodendrocytes with $76.10 \% \pm 2.87 \%$ for miPS-NPCs and $61.88 \%$ $\pm 4.22 \%$ for $\mathrm{mE}-\mathrm{NPCs}$ in response to demyelination (Figure $4 \mathrm{C}$ ).

miPS-NPCs extensively migrate and remyelinate the demyelinated adult spinal cord. As migration of exogenous cells is mandatory for their efficient colonization and remyelination of axons in far-distant lesions, the potential of migration and differentiation into myelin-forming cells of the grafted cells was extensively investigated on serially cut sections. 

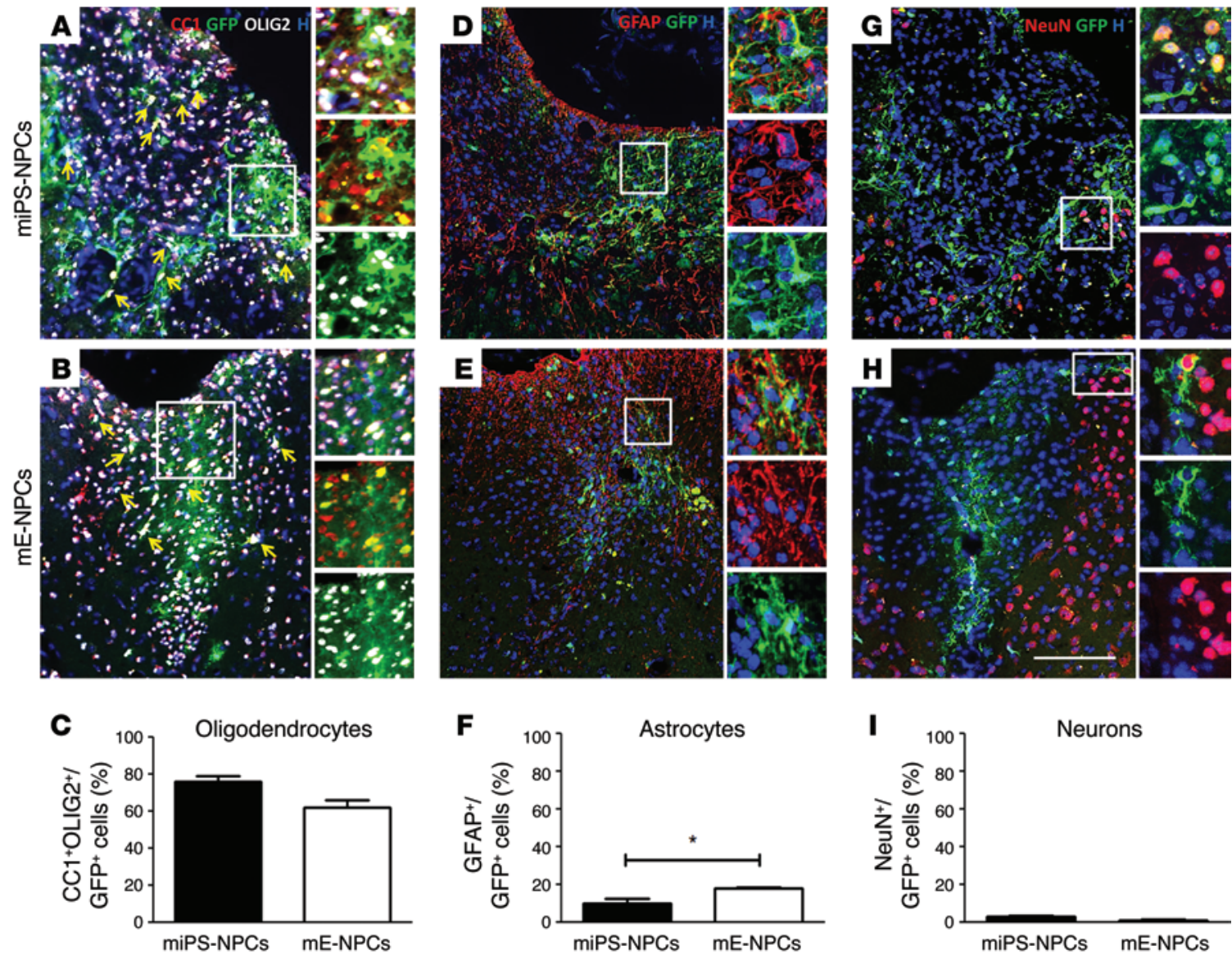

Figure 4. miPS-NPCs show multipotency following transplantation in adult demyelinated Shi/Shi Rag2-/- spinal cord. (A, B, D, E, C, H) Immunolabeling of coronal sections of the grafted Shi/Shi Rag2 $2^{-1-}$ spinal cord 6 wpg. (C, F, I) Quantifications of immunolabed transplanted cells. (A-C) miPS-NPCs (A) and mENPCs (B) generated mainly GFP+ mature oligodendrocytes expressing OLIG2 (white) and CC1 (red). No significant differences in the number of CC1 $/ \mathrm{OLIC2}^{+}$cells were found (C). (D-F) Both cell types differentiated into GFAP ${ }^{+}\left(\right.$red)/GFP ${ }^{+}$(green) astrocytes ( $\mathbf{D}$ and $\mathbf{E}$ ); more GFAP astrocytes were found following engraftment of mE-NPCs than miPS-NPCs (F). (G-I) Few NeuN ${ }^{+}$(red)/GFP+ (green) neurons (about $3 \%$ of total cells) were found in the miPS-NPCs (G) and mE-NPCs (H) grafted mice. No significant difference was observed in neuronal differentiation of miPS- versus embryonic-derived cells (I). Arrow points to triple-labeled cells. Student's $t$ test was used for the statistical analysis of these experiments $\left(n=3-4\right.$ mice per group). ${ }^{*} P<0.05$. H, Hoechst dye. Scale bar: $100 \mu \mathrm{m}$.

First, we evaluated the longitudinal distance in which the $\mathrm{GFP}^{+}$cells distributed in the dorsal funiculus of lesioned Shi/ Shi Rag2 $2^{--}$(Figure 5, A-C) and nude mice (Figure 5D and Supplemental Figure 6, A-E). In the lesioned nude mice, the distribution of miPS-derived cells increased gradually over time, with cell dispersion extending along the dorsal funiculus, covering respectively $6,280 \pm 1,574 \mu \mathrm{m} ; 8,467 \pm 972.2 \mu \mathrm{m}$; and 13,600 \pm $637.9 \mu \mathrm{m}$ at $1 \mathrm{wpg}, 2 \mathrm{wpg}$, and $6 \mathrm{wpg}$, respectively. To take into account the initial mechanical distribution resulting from injection, we grafted the same amount of cells in the dorsal funiculus of nonlesioned mice and evaluated their distance of migration 6 wpg. In these mice, GFP cells distributed over 2,993 $\pm 550.7 \mu \mathrm{m}$ (Supplemental Figure 6E and Figure 5D) and, thus, 4 times less than in demyelinated animals.

In the lesioned Shi/Shi Rag2 $2^{--}$mice, miPS-NPCs distributed over 6,880 $\pm 1,482 \mu \mathrm{m}$ along the dorsal funiculus (Figure 5 , A and C) and mE-NPCs covered $6,440 \pm 1,854 \mu \mathrm{m}$ with a similar migration pattern to miPS cells (Figure 5, B and C). We expected that migration into the Shi/Shi Rag2 $^{--}$environment would be facilitated by the absence of compact myelin. This was not the case, as miPS-NPCs migrated significantly less in Shi/Shi Rag2-- at 6 wpg than in nude mice (Supplemental Figure 6F). Interestingly, the less-efficient migration of miPS-NPCs in Shi/Shi Rag2 ${ }^{-/}$mice was inversely correlated to their higher propensity to differentiate into oligodendrocytes in Shi/Shi Rag2 ${ }^{-/}$compared with nude mice (Supplemental Figure 6G).

We next wondered whether, after successful migration, cells would differentiate into myelin-forming cells and whether this potential would be similar according to the cell source and their environment. Interestingly, coronal and longitudinal sections showed that miPS-NPCs and mE-NPCs extensively remyelinated the demyelinated spinal cord funiculus, as confirmed by the wide expression of MBP in the adult MBP-deficient Shi/Shi Rag2-/ mice (Figure 5, E-K). miPS-NPC- and mE-NPC-derived oligodendrocytes harbored a typical morphology of bona fide myelin-forming oligodendrocytes with several T-shape ending processes (Figure 5 , I and J). We then quantified the extent of remyelination based on MBP expression on coronal sections in Shi/Shi Rag2-- mice. Based on $\mathrm{MBP}^{+}$area, miPS-NPCs were able to produce $\mathrm{MPB}^{+}$ myelin to the same extent of mE-NPCs with 6,200 $\pm 1,582 \mu \mathrm{m}$ and 

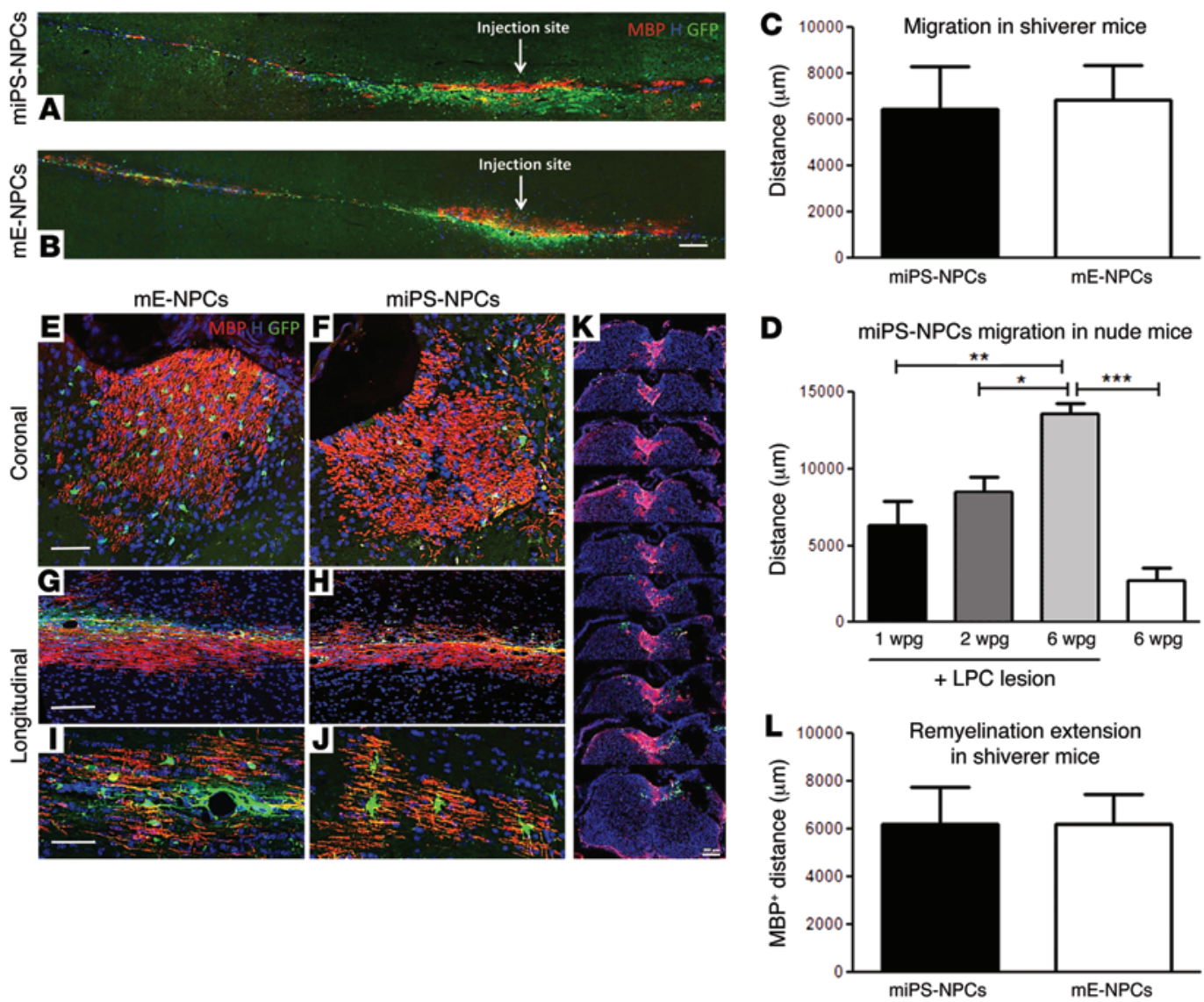

Figure 5. miPS-derived cells extensively migrate and remyelinate the demyelinated adult spinal cord. Combined detection of MBP (red) and GFP (green) in the Shi/Shi Rag2 ${ }^{-1-}$ spinal cord 6 wpg. (A and B) General views of longitudinal sections of mice grafted with miPS-NPCs (A) and mE-NPCs (B), $n=2$ mice per each cell type and per time point (6 and 10 wpg). (C) Evaluation of GFP+ cell dispersion on serial coronal sections. No significant difference in the migration potential of miPS-NPCs and mE-NPCs. (D) Progressive migration of miPS-NPCs in nude mice. (E-H) Coronal (E and F) and longitudinal ( $\mathbf{G}$ and $\mathbf{H}$ ) sections of the grafted MBP-deficient spinal cord illustrating the extent of exogenous myelin 6 wpg. (I and $\mathbf{J}$ ) Views of representative $\mathrm{MPB}^{+}$mature oligodendrocytes derived from miPS-NPCs (I) and mE-NPCs (J). (K) Serial coronal sections depict the widespread distribution of miPS-NPC-derived myelin along the rostro-caudal axis. (L) Quantification of $\mathrm{MBP}^{+}$area on total $\mathrm{MBP}^{+}$coronal sections indicates a perfect overlap between the extent of migration (C) and remyelination by exogenous cells. No obvious difference was observed between miPS-NPCs and mE-NPCs. ${ }^{*} P<0.05$, ${ }^{*} P<0.01$, and ${ }^{* * *} P<0.001$. Student's $t$ test was used for graphs $\mathbf{C}$ and $\mathbf{L}(n=3-4$ mice per group). One-way analysis of variance plus Tukey's multiple comparison tests were used for graph $\mathbf{D}$ ( $n=3-4$ mice per group). H, Hoechst dye. Scale bars: $200 \mu \mathrm{m}$ in $\mathbf{A}$ and $\mathbf{B} ; 50 \mu \mathrm{m}$ in $\mathbf{E}, \mathbf{F}, \mathbf{I}$, and $\mathbf{J}$; and $100 \mu \mathrm{m}$ in $\mathbf{G}$ and $\mathbf{H}$.

$6,200 \pm 1,240 \mu \mathrm{m}$ for miPS-NPC and mE-NPC, respectively (Figure $5 \mathrm{~L}$ ), indicating that migration and remyelination were tightly correlated (Figure 5, A and B). Furthermore, $\mathrm{GFP}^{+}$cells did not express protein zero (P0), a major protein of peripheral myelin, indicating their entire commitment to CNS myelin-forming cells (Mozafari et al., unpublished observations).

To investigate the degree of competition between exogenous and endogenous cells, we evaluated the ratio of graft-derived myelin $\left(\mathrm{MBP}^{+}\right.$area) over total myelin $\left(\mathrm{MOG}^{+}\right.$area) on coronal sections. No significant difference was observed between miPSNPCs and mE-NPCs. miPS-NPC-derived myelin (Figure 6, A-D) extended over $53.12 \% \pm 5.55 \%$ of the whole dorsal funiculus, with $64.13 \% \pm 4.37 \%$ at the injection site. $\mathrm{mE}-\mathrm{NPC}$-derived myelin covered $57.97 \% \pm 9.98 \%$ of the whole dorsal funiculus (Figure 6 , $\mathrm{E}-\mathrm{H}$, and I), with $77.36 \% \pm 5.82 \%$ at the injection site. When $\mathrm{MBP}^{+}$ area was reported to the number of GFP cells (Figure 6J), individual miPS-NPCs generated slightly larger domains of myelination $\left(1,410 \pm 313.6 \mu \mathrm{m}^{2}\right)$ than mE-NPCs $\left(736.4 \pm 8.72 \mu \mathrm{m}^{2}\right)$.
miPS-NPC-derived myelin wraps around host axons, restores nodes of Ranvier, and forms structurally compact myelin following transplantation in the adult demyelinated spinal cord. Next, we investigated the structural characteristics of the miPS-derived myelin in Shi/Shi Rag2-- mice. Longitudinal and coronal sections of the Shi/Shi Rag2-- spinal cord showed that $\mathrm{MBP}^{+}$myelin derived from miPS cells surrounded $\mathrm{NF}^{2} \mathrm{OO}^{+}$host axons as extensively as embryonic cells (Figure 7, A-F). Quantification on confocal images of the proportion of $\mathrm{NF}^{+}$axons surrounded by $\mathrm{MBP}^{+}$myelin at the level of maximal demyelination indicated that $66.69 \% \pm 1.47 \%$ and $71.16 \% \pm 2.75 \%$ of the axons were remyelinated by miPSNPCs and mE-NPCs, respectively (Figure 7M). Furthermore, staining for the paranodal marker CASPR revealed the presence of paranodes in the majority of $\mathrm{MBP}^{+}$myelin structures, confirming the functional integration of miPS-NPC-derived myelin into axoglial elements of the nodes of Ranvier (Figure 7, G, I, and J) with no apparent difference from mE-NPCs (Figure 7, H, K, and L). Our data 6 weeks after transplantation of miPS-NPCs in demyelinated 

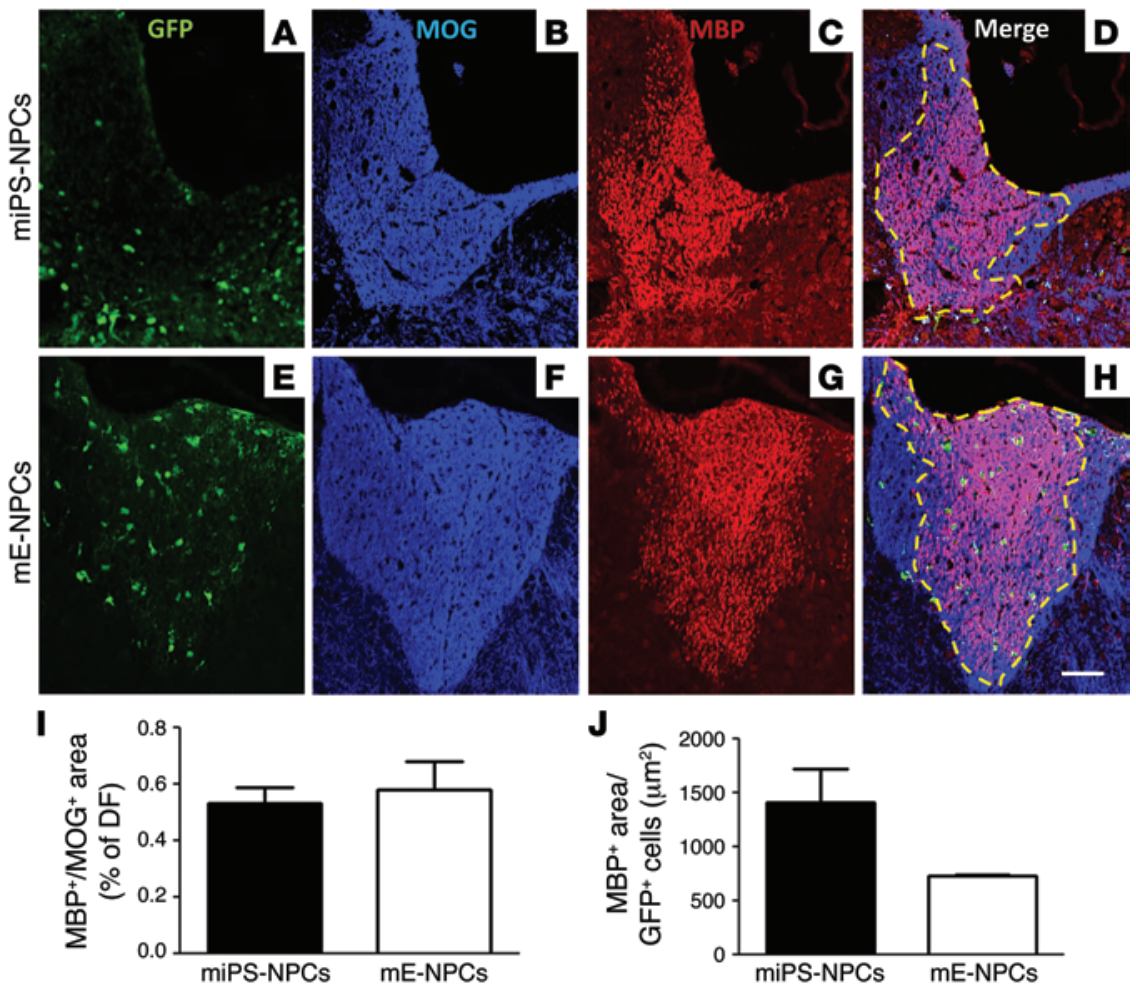

Figure 6. Competitive remyelination potential of iPS-derived cells in demyelinated Shi/Shi Rag2 $^{-1-}$ mice. (A-H) Colabeling of MOC (blue) to detect whole myelin with MBP (red) to detect myelin derived from exogenous cells on coronal sections of demyelinated Shi/Shi Rag2 $2^{-/-}$spinal cord grafted with miPS-NPCs (A-D) and mE-NPCs (E-H) reveals extensive remyelination by exogenous cells compared with endogenous cells. (I) No statistical difference between miPS-NPC- and mE-NPC-derived myelin was detected 6 wpg. (J) When the amount of myelin was expressed per number of $\mathrm{GFP}^{+}$cells in each transplanted group, the myelination domain of miPS-NPCs was greater than that of mE-NPCs, but not statistically different. Dotted lines represent the $\mathrm{MOC}^{+} / \mathrm{MBP}^{+}-$labeled area. Mann Whitney test was used for statistical analysis $(n=3-4$ mice per group). Scale bar: $50 \mu \mathrm{m}$. myelin WT nude mice confirmed similar oligodendroglial differentiation (Supplemental Figure 7A) and remyelination (Supplemental Figure 7B) of the grafted cells. Indeed, $\mathrm{GFP}^{+}$miPS-NPCs produced $\mathrm{MBP}^{+}$myelin around host axons and integrated into axo-glial elements colabeled with the paranodal marker CASPR (Supplemental Figure 7C).

Then, we validated the remyelination potential of the grafted miPS-derived cells by electron microscopy. Exogenous, multilayered, compact myelin generated by either miPS-NPCs (Figure 8, I-L) or mE-NPCs (Figure 8, M-P) surrounded a large proportion of Shi/Shi Rag2 ${ }^{--}$axons compared with the noncompact myelin produced by host cells in intact (Figure 8, A-D) or medium-injected demyelinated Shi/Shi Rag2-- mice (Figure 8, E-H). Quantification of $g$ ratio validated that axons remyelinated by miPS-derived cells (g ratio: $0.789 \pm 0.008$ ) or $\mathrm{mE}-\mathrm{NPCs}$ (g ratio: $0.791 \pm 0.006$ ) have significantly thicker myelin than axons remyelinated by Shi/ Shi Rag2-/- endogenous cells (g ratio: $0.96 \pm 0.003$ ) (Figure 8Q). Furthermore, no correlation was found between the exogenous myelin thickness and axonal diameter (with an approximate thickness of $100 \mathrm{~nm}$ for both miPS-NPCs or mE-NPC-derived myelin). These data demonstrate unambiguously that oligodendrocytes derived from miPS-NPC have the ability to form compact myelin structurally, comparable to that derived from mE-NPCs.

miPS-derived myelin promotes electrophysiological recovery of adult demyelinated Shi/Shi Rag2 $2^{--}$axons. The spinal cord dorsal column contains mainly ascending (afferent) pathways carrying sensory information to the brain. To investigate how the myelin derived from miPS-NPCs influences axonal conduction following demyelination, we measured SSEP in intact Shi/Shi Rag2 ${ }^{-/-}$mice and in age-matched mice injected with $\mathrm{LPC}^{+}$medium or $\mathrm{LPC}^{+}$cells 6 weeks after LPC injection. N1 latency was measured to address the time required in each animal to convey the electrical stimulation from the tibial nerve through the spinal cord toward the recording electrodes on the scull. Latency was delayed in Shi/Shi Rag2 ${ }^{--}$over myelin WT mice $(4,19)$ (Mozafari et al., unpublished observations). In intact and medium-injected Shi/Shi Rag2 $2^{-1-}$ mice, latency was similar with, respectively, $11.79 \pm 1.06 \mathrm{~ms}$ (Figure 9, A and C) and 11.24 $\pm 1.2 \mathrm{~ms}$ (Figure 9, A and D). However, in Shi/Shi $\mathrm{Rag}^{-/-}$mice grafted with miPS-NPCs or mE-NPCs, latencies were reduced nearly 2 -fold, with $6.883 \pm 0.80 \mathrm{~ms}$ and $6.980 \pm 2.07 \mathrm{~ms}$, respectively (Figure 9, A, E, and F). We then estimated conduction velocities for each group. While no major difference in conduction velocities was found in intact $(5.765 \pm 0.57 \mathrm{~m} / \mathrm{s})$ and lesioned Shi $/ \mathrm{Shi}$ Rag2 $^{-/-}$mice treated with medium $(5.957 \pm 0.54 \mathrm{~m} / \mathrm{s})$, the estimated conduction velocities increased up to $9.934 \pm 1.4 \mathrm{~m} / \mathrm{s}$ and $10.96 \pm$ $2.86 \mathrm{~m} / \mathrm{s}$, respectively, in lesioned Shi/Shi Rag2 ${ }^{-/-}$mice grafted with either miPS-NPCs or mE-NPCs (Figure 9B), thus indicating that irrespective of their tissue sources, grafted NPCs have the ability to restore conduction of the dysfunctional shiverer axons.

\section{Discussion}

Adult white-matter tracts in the CNS are highly vulnerable to various types of damages from hypoxic-ischemic, metabolic, or traumatic forms of demyelination to inflammatory and immunemediated insults (20). Remyelination of adult axons in various neuropathological conditions is known to rescue the lost function and protect neurons from subsequent axonal degeneration. The ability to efficiently remyelinate demyelinating insults has been investigated through the development of induced cell reprogramming strategies. Studies focused on generating safe, renewable, and autologous sources of myelin-forming cells. Searching for a quicker or safer method, investigators tried to generate NPCs 

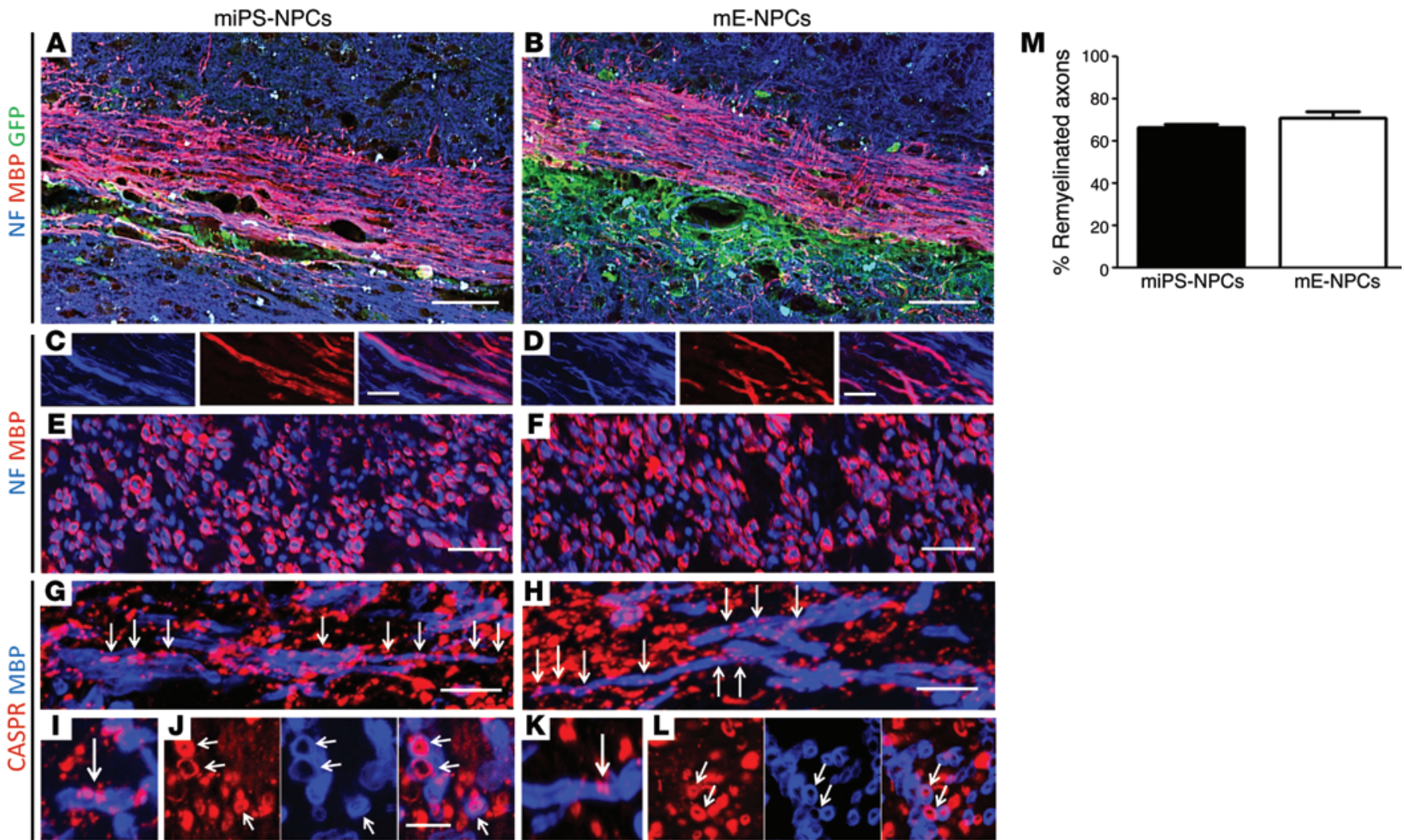

Figure 7. miPS-derived myelin wrap around numerous demyelinated Shi/Shi Rag2-/- axons and restore nodes of Ranvier. (A-D) Longitudinal sections illustrating extensive colabeling of $\mathrm{MBP}^{+}$myelin (red) derived from miPS (A) or mE-NPCs (B) with NF200 ${ }^{+}$axons (blue). (C and D) Higher magnification of $\mathbf{A}$ and $\mathbf{B}$. (E and $\mathbf{F}$ ) Coronal sections at high magnification confirm ensheathment of exogenous $\mathrm{MBP}^{+}$myelin around NF200+ axons. (G and $\mathbf{H}$ ) Longitudinal sections showing restoration and alignment of several nodes of Ranvier, identified by codetection of paranodal CASPR (red) associated with $\mathrm{MBP}^{+}$myelin (blue) derived from miPS (G) and mE-NPCs (H). (I and $\left.\mathbf{L}\right)$ Higher magnification of nodes of Ranvier in longitudinal sections (I and $\mathbf{K}$ ) and coronal sections (J and $\mathbf{L}$ ) illustrating colabeling of MBP+ myelin (blue) and CASPR proteins (red) for miPS-NPCs (G, I, and J) and mE-NPCs (H, $\mathbf{K}$, and $\mathbf{L}$ ). (M) Quantification on coronal sections stained for NF and MBP indicate that the grafted cells successfully remyelinate host axons with no significant difference between miPS- or embryonic-derived cells. One-way analysis of variance plus Tukey's multiple comparison tests were used for graph $\mathbf{M}$ ( $n=3-4$ mice per group). $n=2$ mice per cell type and per time point ( 6 and $10 \mathrm{wpg}$ ) for longitudinal sections; $n=3-4$ for coronal sections. Scale bars: $50 \mu \mathrm{m}$ in $\mathbf{A}$ and $\mathbf{B} ; 10 \mu \mathrm{m}$ in $\mathbf{C}-\mathbf{F}$; and $5 \mu \mathrm{m}$ in $\mathbf{G}-\mathbf{L}$.

or OPCs directly reprogrammed from somatic cells. However, in doing so, they largely lost the reprogramming efficacy, cell expandability/renewability $(9,14)$, or the competence to generate myelin-forming cells in vitro or in vivo $(11,13-15,21-23)$. It has been reported that reprogramming of fibroblasts to NPCs could be achieved using a single transcription factor, SOX2 (11). Moreover, SOX2 alone was sufficient for in vivo reprogramming of astrocytes to neuroblasts (24). Here, we used (i) Sox $2 \beta G e o$ knockin murine cells (25) to confer cell purity and safety, (ii) indirect reprogramming to ensure efficient cell renewability, and (iii) conversion of iPS into NPCs to provide efficient migration $(2,26)$, genesis of oligodendrocytes $(2,3)$, and neural protection $(18,27)$. We demonstrate the impressive survival, colonization, and remyelination potential of Sox $2 \beta G e o$ iPS-NPCs after a single injection in the adult demyelinated spinal cord, suggesting that deriving NPCs from induced pluripotent cells is a promising step toward the use of patients' own cells in myelin diseases of the adult CNS.

miPS-NPCs downregulate proliferation and do not form tumors in adult mice. In the present study, Sox $2 \beta G e o$ murine fibroblasts were reprogrammed by a OCT4, SOX2, and KLF4 transcription- factor cocktail, lacking c-MYC, a factor causing tumorogenesis following transplantation of iPSC-NPCs $(28,29)$. Selection of cells expressing the neuroepithelial marker SOX2 warranted additional measures to ensure the safety of the strategy.

We show that the miPS-NPC proliferation rate was not significantly different in vitro from $\mathrm{mE}$-NPCs and was timely downregulated after transplantation. These observations were consistent with the absence of tumor formation at histochemical and ultrastructural levels and indicate that, within the experimental time frame, Sox $2 \beta$ Geo miPS-NPCs did not divert into uncontrolled proliferating cells in adult nude or in Shi/Shi Rag2/mice strains. Moreover, negative expression of pluripotency and tumorogenesis markers OCT4 and NANOG in vitro (18) was confirmed in vivo at different time points following miPS-NPCs engraftments ( 6 and $10 \mathrm{wpg}$ ) in adult mice. Although tumor formation after long-term transplantation was not investigated in the present study, the extensive differentiation of miPS-NPCs into functional myelin-competent oligodendrocytes forming nodal structures and restoring axonal conduction fully sustains their complete integration in their host parenchyma. 

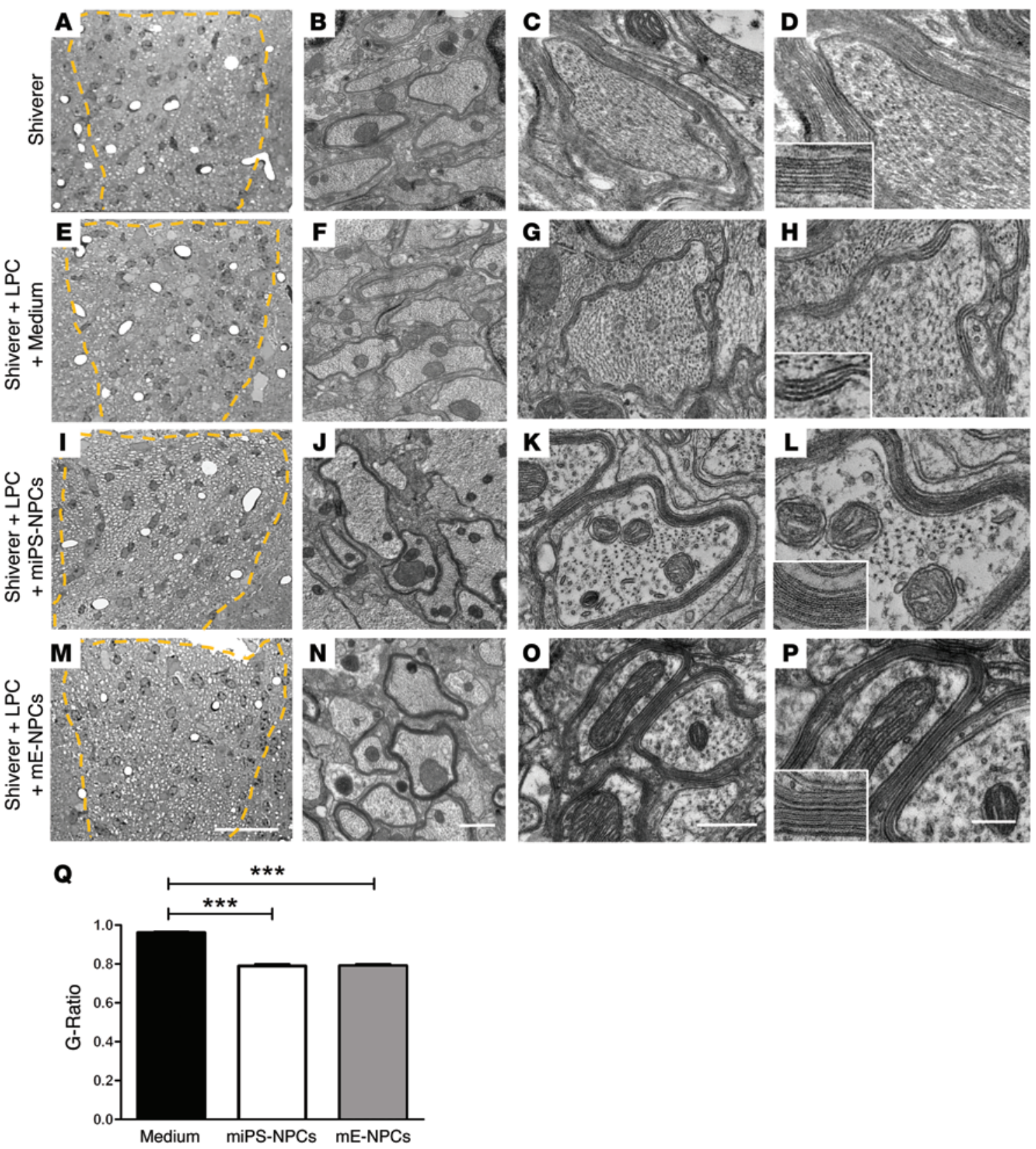

Figure 8. miPS-derived oligodendrocytes produce compact myelin in the demyelinated Shi/Shi Rag2 ${ }^{-/-}$spinal cord. (A-P) Ultrastructure of myelin on coronal sections of the dorsal funiculus (A-D) of intact Shi/Shi Rag2-/- mouse spinal cord; (E-H) 6 weeks after LPC-induced demyelination followed by medium injection; (I-P) 6 weeks after LPC injection and engraftment of miPS-NPCs (J-L) and mE-NPCs (M-P). While Shi/Shi Rag2 $2^{-/-}$axons were surrounded by wraps of loose uncompacted myelin in normal or remyelinated (with fewer warps) conditions, they were surrounded by thick and compact normal myelin derived from the grafted NPCs (identified at G-L for miPS-NPCs and N-P for mE-NPCs). No difference in compaction and structure is observed between myelin derived from miPS-NPCs and mE-NPCs. (Q) Quantification of $g$ ratio revealed a significant difference between myelin thickness of axons remyelinated by endogenous cells versus that of axons remyelinated by miPS-NPCs or mE-NPCs. Dotted lines in A, E, I, and $\mathbf{M}$ represent the lesion site in the dorsal funiculus. One-way analysis of variance plus Tukey's multiple comparison tests were used for graph $\mathbf{Q}(n=3-4$ mice per group). Insets in $\mathrm{D}, \mathrm{H}, \mathrm{L}$, and $\mathrm{P}$ are enlargements of myelin, illustrating its fine structure. ${ }^{* *} P<0.001$. Scale bars: $30 \mu \mathrm{m}$ in $\mathbf{A}, \mathbf{E}, \mathbf{I}$, and $\mathbf{M} ; 1 \mu \mathrm{m}$ in $\mathbf{B}, \mathbf{F}, \mathbf{G}$, and $\mathbf{N} ; 500 \mathrm{~nm}$ in $\mathbf{C}, \mathbf{G}, \mathbf{K}$, and $\mathbf{0}$; and $200 \mathrm{~nm}$ in $\mathbf{D}, \mathbf{H}, \mathbf{L}$, and $\mathbf{P}$.

miPS-NPCs behave as bona fide multipotent NPCs in adult mice. While most studies compared the behavior of miPS-NPCs with that of embryonic stem cell-derived (ES-derived) NPCs, we chose to compare miPS-NPCs, in vitro and in vivo, with NPCs derived from embryonic mouse brain - which are physiologically committed to neural fate - with the aim to investigate whether induced pluripotent-derived NPCs behave as efficiently as physiologically committed cells. Lesion size and transplanted cell density were carefully controlled, and their evaluation ruled out the influence of potential differences in these 2 paradigms in comparing the transplanted groups. Data highlighted only subtle differences between the 2 cell sources. miPS-NPCs expressed markers associated with immature neural cells in the absence of those related with more mature differentiation stages in vitro. Although very 
A

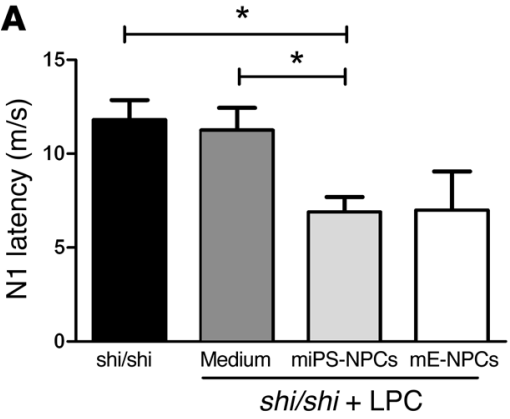

C

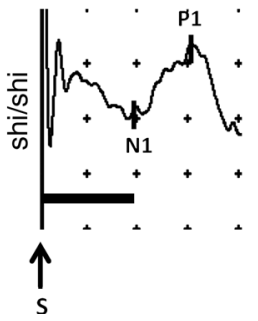

D

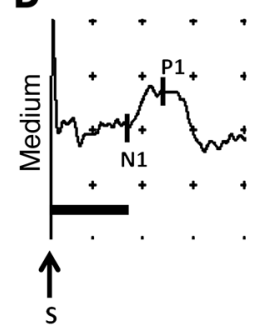

B

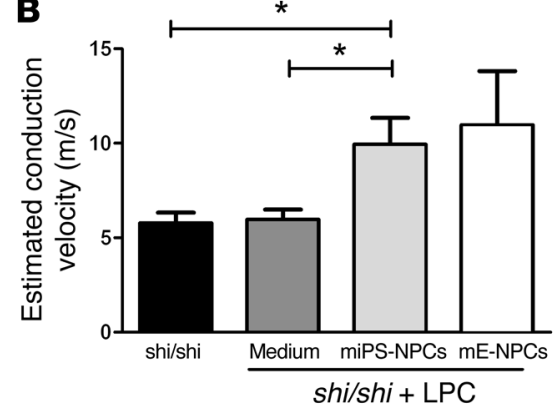

E

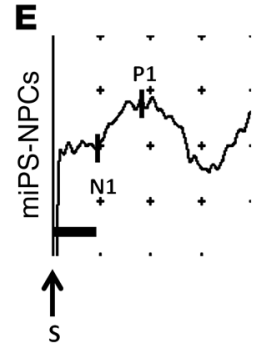

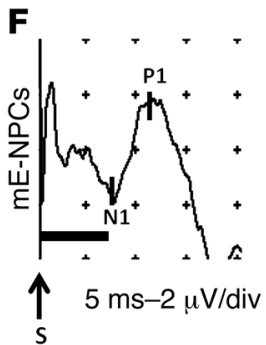

Figure 9. miPS-derived myelin improves conduction of the dysfunctional Shi/Shi Rag2 ${ }^{-/-}$axons. (A) N1 latency was measured following SSEP in different groups of Shi/Shi Rag2 ${ }^{--}$mice 6 wpg or medium injection. miPS-derived myelin significantly decreased conduction latency in intact $(P=0.01)$ or demyelinated spinal cord injected with medium $(P=0.02)$. (B) Evaluation of axonal conduction velocity in the different groups. While no difference was detected in demyelinated and medium-treated mice over intact mice, grafted animals showed a significant increase in conduction velocities over intact $(P=0.03)$ or demyelinated and medium-treated mice $(P=0.02)$. $(\mathbf{C}-\mathbf{F})$ Representative SSEP profiles for intact (C), and lesioned mice injected with medium (D), miPSNPCs (E), mE-NPCs (F), and grafted mice ( $n=4-6$ mice per group). Mann Whitney test was used for the statistical analysis. S, Stimulation. ${ }^{*} P<0.05$. Scales in profiles: $5 \mathrm{~ms}$ (horizontal) or $2 \mu \mathrm{V}$ (vertical) per divisions. similar results were achieved with mE-NPCs, the latter seemed to be slightly more engaged into astroglial differentiation in vitro and in vivo. Irrespective of this minor difference, both cell types were equally competent in generating oligodendrocytes and neurons. Thus, both cell sources were tripotent, given their ability to generate the 3 major neural cell types.

Grafted miPS-NPCs gave rise to $10 \%$ of astrocytes compared with $20 \%$ for embryonic cells. Although astrocytes could prevent remyelination contributing to the glial scar or promote (re)-myelination, enhancing the differentiation of endogenous OPCs by trophic factors (30), our data indicate that genesis of 2-fold more astrocytes by mE-NPCs did not have a major impact on (re)-myelination, as both cell sources were equally myelin competent. Moreover, grafted NPCs did not seem to trigger more astrogliosis, comparing grafted and nongrafted mice (Mozafari et al., unpublished observation). Interestingly, similar amounts of astrocytes were generated by grafted adult murine NPCs (19) while about 30\%$40 \%$ of astrocytes were generated after engraftment of human NPCs (2). OPCs are also candidates of interest for cell therapy. These cells are bipotential in vivo, generating astrocytes in addition to oligodendrocytes. Goldman's team reported less than 3\% of astrocytes were generated by mouse OPCs transplantated in the newborn shiverer corpus callosum compared with $10 \%-20 \%$ by grafted human fetal OPCs (5) or human iPS-OPCs (hiPS-OPCs) (8). Efficient (re)-myelination was observed in all of these studies, regardless of the different animal models used, which indicated no clear difference in astrocyte differentiation potential between the 2 cell types. While both NPCs and OPCs can generate multiple cell types (31), including myelin-forming oligodendrocytes and astrocytes, NPCs provide trophic support and immunomodulation (1), properties that were not yet reported for OPCs. It remains an open question whether one of these cell types (NPCs/OPCs) has a clear therapeutic advantage over the other for future transplantation strategies for myelin disorders - a question that should be addressed only comparing age-matched populations grafted in the same disease context.
Furthermore, our studies showed almost none or very limited neuronal derivation of NPCs (fetal human cells $\sim 0.4 \%-0.7 \%$ and mouse cells $\sim 1-2 \%$; ref. 2 and the current study) when grafted in the "adult demyelination" context. This is not surprising, as cues for neuronal replacement are not available in the LPC model in which demyelination occurs in the absence of neuronal/axonal loss. Whether neuronal differentiation potential of the grafted cells can help or adverse the clinical conditions still remains to be addressed in a model with demyelinating lesions containing or leading to neuronal loss.

miPS-NPCs timely differentiate into functional oligodendrocytes. In LPC-induced demyelination, axons are remyelinated by endogenous myelin-competent cells, oligodendrocytes, and Schwann cells (32). There is an onset of remyelination the first week after demyelination; the process is nearly completed by 28 days (33). We show that miPS-NPCs differentiated timely into bona fide mature myelinating oligodendrocytes. While NPCs (nestin ${ }^{+}$), and OPCs $\left(\mathrm{OLIG}^{+} / \mathrm{CC1}^{-}\right)$decreased with time, the number of mature oligodendrocytes $\left(\mathrm{OLIG}^{+} / \mathrm{CC}^{+}\right)$increased 2 - and 4 -fold at $2 \mathrm{wpg}$ and 6 wpg respectively, suggesting their competitive maturation with endogenous oligodendroglial cells. Moreover, exogenous miPSNPCs produced extensive myelin within the timeframe of endogenous remyelination and with no obvious difference between $\mathrm{mE}$ NPCs and miPS-NPCs. Similar observations were made for adult mouse NPCs 6 wpg in the adult shiverer spinal cord (19). This is in contrast to NPCs derived from human tissue, which differentiate along a much slower process when grafted in the same experimental paradigm (2). Thus, environmental cues of the adult demyelinated CNS timely regulate the differentiation of rodent NPCs into oligodendrocytes, irrespective of their age (adult or embryonic), source (multipotent or pluripotent), and primary origin (brain or skin fibroblasts); rather, the process is species specific.

miPS-NPCs outcompete endogenous cells to restore myelin structure and function in the adult demyelinated spinal cord. Assessment of global myelin (endogenous and exogenous) revealed by MOG staining and donor-derived myelin identified by MBP indi- 
cated that, in the lesion core, $65 \%$ (up to $66 \%$ of the axons) of the dorsal funiculus area was remyelinated by miPS-derived cells against $35 \%$ by endogenous cells (encompassing both existing and newly formed myelin). Moreover, electron microscopy analysis indicated that miPS-derived oligodendrocytes generated structurally normal compact myelin around host axons. Evaluation of $g$ ratios showed that, for comparable axon diameters, exogenous myelin appeared clearly thicker than developmental $(4,14)$ or newly formed shiverer myelin. Interestingly, normal endogenous or neonatally grafted cells have the opportunity to adjust the correlation between axon diameter and myelin thickness or length during the CNS development $(4,34)$. However, unlike these observations, we found that miPS-NPCs and $\mathrm{mE}-\mathrm{N}$ PCs grafted in the adult demyelinated context do not respect this developmental rule, since they produced similar amounts of multilayered and compact myelin $(\sim 100 \mathrm{~nm})$ around adult host axons, irrespective of their diameters. Engraftment of adult NPCs in adult mice produced similar amounts of myelin around host axons (19). This indicates that, in the adult demyelinated CNS, unlike during development, myelin thickness is not controlled by axonal signaling (35) but is an intrinsic property of the myelin-competent cell.

Uncompacted myelin, as observed in shiverer mice, results in abnormal saltatory conduction compared with myelin WT mice $(4,19)$ (Mozafari et al., unpublished observations). We used SSEP recordings to investigate the impact of the grafted cells on the shiverer axonal dysfunction. Difference in conduction velocity or latency responses in intact versus demyelinated shiverer mice treated with medium were not detected. This was predictable, since spontaneous remyelination restores the demyelination-induced altered electrophysiological function (36-38). However, engraftment of miPS-derived cells decreased the latency response and consequently increased axonal conduction velocity of the adult shiverer axon, as previously observed in vitro (39) and in vivo (19) for primary glial or neural progenitors. Moreover, the correlation between the electrophysiological and ultrastructural data strongly suggest that the functional benefit provided by the grafted cells results from the thick and compact myelin generated by the immature exogenous cells compared with the thin and noncompacted myelin of endogenous cells.

The benefit of exogenous primary OPCs over endogenous cells in repopulating demyelinated lesions in WT rats was previously suggested (40). However, in the absence of a tracking system to visualize the grafted cells and their myelin, evidence for competitive remyelination was indirect and conclusions remained elusive. A competitive myelinating advantage of neonatally engrafted human glial progenitor cells over mouse endogenous shiverer cells was also reported (5). These experiments showed that oligodendrocyte differentiation of neonatally administrated human cells operates in a context-dependent fashion and suggested that human cells are more competent than rodent cells. Our data obtained in the adult demyelinated spinal cord, a far less permissive environment than the developing brain, implement these observations as grafted NPCs migrate less but differentiate more into oligodendrocyte in myelin-deficient mice than in WT nude mice. Additionally, we provide overwhelming evidences that skin-derived NPCs are as competent in adult spinal cord repair as brain-derived NPCs. Finally, our work indicates that improved competitivity is not true only for human cells, since mouse cells outcompete endogenous cells efficiently.

Conclusion. Wang et al. showed the impressive myelination achieved by multiple injections of human iPSC-derived OPCs when grafted in the developing newborn dysmyelinated shiverer forebrain (8). Here, we convincingly demonstrate that miPS-derived NPCs delivered by a single injection in the demyelinated adult spinal cord invade as efficiently as brain-derived NPCs, a large portion of the adult neurax, and generate an impressive proportion of functional remyelinating oligodendrocytes, thus indicating the high efficiency of iPS-derived NPCs - even when not manipulated in vitro - to be committed to the oligodendrocyte lineage. iPS-NPCs also generate astrocytes, which could contribute positively to the repair events. These observations highlight the great differentiation plasticity of these cells and their capacity to adapt to the environment in which they are placed. This, added to the recently established neuroprotective properties of NPCs derived from pluripotent cells (18), opens great promise for their use in regenerative medicine for myelin disorders of the adult CNS.

\section{Methods}

\section{Cell culture}

Mouse iPS-NPCs. miPS cells were generated from reprogrammed E14.5 C57Bl/6 Sox $2^{\mathrm{Bgeo} /+}$ knockin embryonic fibroblasts expressing the $\beta g e o$ gene under the control of Sox 2 promoter that gives cell resistance to neomycin (18). NPCs were grown on an uncoated plastic flask (T75) in Euromed-N (EuroClone) supplemented with 1\% N2 supplement (Invitrogen), Pen/strep (Invitrogen), and L-glutamine (200 nM) (Invitrogen), as well as $20 \mathrm{ng} / \mathrm{ml}$ basic fibroblast growth factor (bFGF) (PeproTech) and $20 \mathrm{ng} / \mathrm{ml}$ epidermal growth factor (EGF) (PeproTech). Fresh medium was added every 2 days. Cells were split once a week depending on cell density and reseeded $10^{6}$ cells/T75 flask in 10 ml of medium.

mE-NPCs. Primary mouse NPCs were obtained from E12/E13 $\mathrm{C} 57 \mathrm{Bl} / 6$ mice as previously described (41). Briefly, brains were dissected free of meninges, dissociated using ATV (0.05\% trypsin, 0.1\% glucose, and 0.5 nM EDTA). Collected cells were resuspended in NEF medium composed of DMEM/F12 medium (1:1) supplemented with N2 supplements (1\%), B27 (0.5\%), insulin ( $25 \mu \mathrm{g} / \mathrm{ml})$, glucose (6 mg/ $\mathrm{ml})$, Hepes $(5 \mathrm{mM})$, FGF2 (20 ng/ml), and EGF $(20 \mathrm{ng} / \mathrm{ml})$. NPCs were dissociated once a week and reseeded at the density of $10^{6}$ cells/ T75 flask in $10 \mathrm{ml}$ of medium.

To allow tracing of cells for in vivo studies, cells were transduced with a third-generation lentiviral vector encoding the GFP as a tracer gene. More than $80 \%$ of the cells were labeled with this method (18).

Immunocytochemistry. Cell characterization was performed on dissociated $\mathrm{GFP}^{+}$miPS-NPCs and mE-NPCs, seeded on 4-well plastic plates $\left(2 \times 10^{4}\right.$ cells/well; duplicate wells for each cell marker) already coated with polyornithine and laminin (Sigma-Aldrich). Cells were fixed in $4 \%$ paraformaldehyde (Sigma-Aldrich) for 10 minutes prior to immunostaining for markers of mature or immature neural cells. The following antibodies were used for cell characterization: anti-nestin (MAB353, Millipore), anti-Ki67 (556003, BD Biosciences), anti-OLIG2 (AB9610, Millipore), anti-vimentin 


\section{Table 1. Primers}

\begin{tabular}{|c|c|c|c|}
\hline mRNA & Sense $\left(5^{\prime}-3^{\prime}\right)$ & Antisense $\left(5^{\prime}-3^{\prime}\right)$ & Size (bp) \\
\hline Nestin & CAGAGAGGCGCTGGAACAGAG & AGACATAGGTGGGATCGGAGT & 465 \\
\hline Sox2 & AGAACCCCAAGATGCACAAC & ATGTAGGTCTGCGAGCTGGT & 456 \\
\hline Sox9 & CAAGTGTGTGTGCCGTGGATAG & CCAGCCACAGCAGTGAGTAAGAA & 100 \\
\hline Musashi & GGCTTCGTCACTTTCATGGACC & GGGAACTGGTAGGTGTAACCAG & 541 \\
\hline Olig2 & TAGTTTCGCGCCAGCAGCAG & GGCGGTGGCTTCAAGTCATC & 259 \\
\hline Olig1 & AAGGAGGACATTTCCAGACTT & GCTCTCTAAACAGGTGGGATTCA & 153 \\
\hline$N k \times 2.2$ & ATGTCGCTGACCAACACAAA & GACGGGGAATTCTCGGGAAA & 194 \\
\hline Sox10 & CGGTGAACTGGGCAAGGTCA & AGctctGtctTtGGGGTGGTt & 602 \\
\hline$\beta 3$-tubulin & GGССтССTССтСТСАAСАAGTATGT & СGСССтCTGTATAGTGC & 170 \\
\hline Gfap & GAGATCGССАССТACAGGAA & GСТССТGСТTССАСТССТTA & 199 \\
\hline$\beta$-actin & GATCACGATCGCTGCGCTGGTCG & GCCTGTGGTACGACCAGAGGCA & 437 \\
\hline
\end{tabular}

Sense and antisense primers of markers of immature or mature neural cells used in RT-PCR analysis for characterization of embryonic and miPSderived NPCs.

(V2258, Sigma-Aldrich); anti- $\beta 3$-tubulin (T8660, Sigma-Aldrich); anti-MAP2 (M1406, Sigma-Aldrich); anti-PDGFR $\alpha$ (sc-338, Santa Cruz Biotechnology Inc.), anti-O4 (mouse IgM hybridoma from ATCC), and anti-GFAP (Z0334, Dako). Cells were then incubated with the corresponding secondary antibodies (Jackson ImmunoResearch Laboratories Inc., Invitrogen). Hoechst dye $(1 \mu \mathrm{g} / \mathrm{ml}$, Sigma-Aldrich) was used to stain all cell nuclei.

Gene expression study. RNA extraction was performed using an RNA extraction kit (catalog 74104, QIAGEN) according to the manufacturer's instructions, and followed by treatment with RNase-free DNase Set (catalog 79254). RNA quality was evaluated using Thermo Scientific NanoDrop instrument. cDNA synthesis was performed using 500 ng of total RNA and Thermo Script RT reverse transcriptase (Invitrogen). Control negative experiments were carried out for all samples lacking ThermoScript enzymes (Invitrogen). One microliter of the resulting cDNA was then used for each PCR reaction. PCRs were performed with primers ( $1 \mu \mathrm{M}$ of each) and $12.5 \mu \mathrm{l}$ Green Master Mix (catalog M7112, Promega) with the following reaction cycling parameters: $94^{\circ} \mathrm{C}, 2$ minutes; $94^{\circ} \mathrm{C}, 45$ seconds; $55^{\circ} \mathrm{C}$ (annealing temperature regarded for all genes), 60 seconds; and $72^{\circ} \mathrm{C}, 5$ minutes for 35 cycles. To ensure equal loading of cDNA into RT reactions, $\beta$-actin mRNA was amplified as an internal control. PCR products were resolved by electrophoresis on a caliper LabChip (PerkinElmer). At least 3 independent experiments were performed for every set of RT-PCR analysis. Mouse primer sequences and lengths of the amplified products are listed in Table 1.

\section{Demyelination and cell transplantation}

Mice were housed under standard conditions of 12-hour light/ 12-hour dark cycles with ad libitum access to dry food and water cycle at ICM institute's animal facility. To study the behavior of miPS-NPCs in terms of their safety, migration, and differentiation potentials in adult demyelinating condition, we used 2 different immunodeficient mouse strains: nude with normal myelination and Shi/Shi Rag2-/- with dysmyelination (MBP-deficient) backgrounds.

Nude mice. Adult RjOrl:NMRI-Foxn $1^{n u} /$ Foxn $1^{n u}$ immunodeficient mice (8-9 weeks of age, Janvier) were anesthetized by i.p. injection of a mixture of $100 \mathrm{mg} / \mathrm{kg}$ ketamine (Alcyon) and $10 \mathrm{mg} / \mathrm{kg}$ xylazine (Alcyon). Focal demyelination was performed as previously described $(2,42)$ by stereotaxic injection of $1 \mu \mathrm{l}$ of $1 \%$ lysolecithin (LPC, Sigma-Aldrich) in $0.9 \% \mathrm{NaCl}$ into the dorsal funiculus of the spinal cord at the level of the 13th thoracic vertebrate. Forty-eight hours after demyelination, mice received a single injection $(1 \mu \mathrm{l}$, $\left.10^{5} / \mu \mathrm{l}\right)$ of $\mathrm{GFP}^{+}$miPS-NPCs $(n=15)$ or mE-NPCs $(n=10)$, or the same amount of medium $(n=10)$ at the site of demyelination, as previously described (2). All injections (LPC or cells) were performed at low speed ( $1 \mu \mathrm{l} / 2 \mathrm{~min})$ using a stereotaxic frame equipped with a micromanipulator and a Hamilton syringe. Animals $(n=35)$ were sacrificed 1 and 2 wpg ( $n=5$ for each cell type or medium and for each time point) and $6 \mathrm{wpg}$ ( $n=5$ for miPS-NPCs) by transcardiac perfusion of $1 \times$ PBS and $4 \%$ paraformaldehyde. Five more animals received the same amount of miPS-NPCs in the dorsal funiculus without LPC injection and were sacrificed $6 \mathrm{wpg}$. After dissection, tissues were postfixed in the same fixative for 1 hour and then processed in $20 \%$ sucrose in $1 \times$ PBS overnight. Spinal cords $(\sim 12 \mathrm{~mm}$, including the lesion site at the center) were transversely cut into 3-5 pieces ( $\sim 3 \mathrm{~mm}$ each) and were serially ordered and placed in the small plastic containers, embedded in cryomatrix (Thermo Scientific), frozen in cold isopentane at $-60^{\circ} \mathrm{C}$, and stored at $-20^{\circ} \mathrm{C}$ until use. Transverse sections of $12 \mu \mathrm{m}$ were cut with a cryostat (Leica Microsystems), collected on 3 series of 10 slides each (30-50 sections/slide), and used for IHC.

Shi/Shi Rag2 ${ }^{-/}$mice. Shiverer mice (Mbpshishi, referred to as Shi/ Shi) were crossed to Rag2 null immunodeficient mice (43) to generate a line of Shi/Shi Rag2 $2^{--}$dysmyelinating immunodeficient mice. Mice ( $n=40,8^{-9}$ weeks of age) were demyelinated and transplanted as described above. Twenty more adult Shi/Shi Rag2 $2^{-/}$age-matched mice were demyelinated and received $1 \mu \mathrm{l}$ of medium without cells in their dorsal funiculus. Animals were sacrificed $6 \mathrm{wpg}(n=15$ for each cell type or medium groups) and $10 \mathrm{wpg}$ ( $n=5$ per group) for histochemical analysis and were processed as described above. In addition to transverse sections, 2 animals per each cell type were used for the longitudinal sections ( $12 \mu \mathrm{m}$ thickness) of the spinal cords at 6 and $10 \mathrm{wpg}$.

\section{IHC}

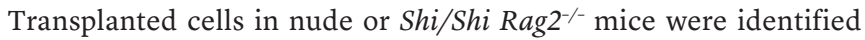
with an antibody directed against GFP (GFP-1020, Aves Labs Inc.). The phenotype of $\mathrm{GFP}^{+}$cells was determined by IHC using the following antibodies: anti-nestin (MAB353, Millipore), anti-Ki67 (556003, BD Biosciences), anti-Olig2 (AB9610, Millipore), antiCC1 (OP80, Millipore), anti-GFAP (Z0334, Dako), anti-PDGFR $\alpha$ (sc-338, Santa Cruz Biotechnology Inc.), anti-NG2 (AB5320, Millipore), anti-NogoA (AB5888, Millipore), anti-SOX10 (sc-17343, Santa Cruz Biotechnology Inc.), anti-NeuN (MAB377, Millipore), anti-MOG (mouse IgG1 hybridoma, clone C18C5; provided by C. Linnington, University of Glasgow, Glasgow, United Kingdom), anti-MBP (AB980, Millipore), anti-NF200 (N0142, Sigma-Aldrich), anti-NF165 (mouse IgG1 hybridoma 2H3, Developmental Studies Hybridoma Bank), anti-CASPR (gift from E. Peles, Rehovot, Israel), anti-caspase3 (9661, Cell Signaling Technology), anti-OCT4 (2840X, Cell Signaling Technology), and anti-NANOG (4903X, Cell Signaling Technology). For MBP and MOG staining, slices were pretreated with ethanol (10 $\mathrm{min}$ at room temperature). For CASPR staining, slices were incubated in methanol $\left(10 \mathrm{~min}\right.$ at $\left.20^{\circ} \mathrm{C}\right)$ and saturated in the presence of $0.1 \%$ glycine (Research Organics Inc.). 
Sections were incubated with the corresponding secondary antibodies from Jackson ImmunoResearch Laboratories Inc. or Alexaconjugated antibodies (Invitrogen) containing Hoechst dye (1 mg/ $\mathrm{ml}$ ). A Carl Zeiss microscope equipped with ApoTome.2 was used for tissue scanning, cell visualization, and imaging.

\section{Electron microscopy}

For electron microscopy, Shi/Shi Rag2 ${ }^{-/-}$mice were perfused 6 wpg or medium injection ( $n=4$ per group), with $1 \%$ PBS followed by a mixture of $4 \%$ paraformaldehyde/2.5\% glutaraldehyde (Electron Microscopy Science) in 1\% PBS for 45 minutes. Two hours after fixation in the same solution, spinal cords were cut in $0.5-1 \mathrm{~mm}$ pieces and fixed in $2 \%$ osmium tetroxide (Sigma-Aldrich) overnight. After dehydration, samples were embedded in Epon. Ultra-thin sections $(80 \mathrm{~nm}$ ) were examined with a HITACHI $120 \mathrm{kV}$ HT-7700 electron microscope.

\section{Somatosensory-evoked potentials}

Electrophysiological recordings of somatosensory-evoked potentials (SSEPs) were performed 6 weeks after injection of LPC or medium in homozygous Shi/Shi Rag2-/- mice, as well as on a group of intact homozygous Shi/Shi Rag2--- mice ( $n=4-6$ per group, 13-14 weeks of age for all animals) as previously described (44-46). Briefly, mice were anesthetized by i.p. injection of ketamine $(100 \mathrm{mg} / \mathrm{kg})$ and xylazine (10 mg/kg) (Alcyon). Body temperature was maintained between $34^{\circ} \mathrm{C}-37^{\circ} \mathrm{C}$ by a feedback-controlled heating blanket (CMA Microdialysis). Constant current stimuli (Amplitude $2.5 \mathrm{~mA}$, pulse $200 \mu \mathrm{s}$, frequency $3.5 \mathrm{~Hz}$ ) were applied to the middle of tibial nerves by s.c. electrodes in hindlimbs and SSEP recorded from electrodes localized over the sensorimotor cortex (MN3512P150, Spesmedica). A ground electrode was placed s.c. over the cervical spine. Recordings were performed by the data acquisition system Neuro-Mep-Micro of Neurosoft, and signals were filtered at 20-2,000 Hz. Each response latency was measured as the time between the onset of stimulus artifact to the first peak (N1) for each animal as the average of 500 sweeps. Conduction velocity was calculated by estimation of the distance between the stimulation and recording electrodes.

\section{Quantification}

Cell characterization in vitro. Each staining was performed on duplicate glass coverslips in 3 independent experiments. For each staining, 10 independent fields including at least 3,000 cells were counted. A Zeiss microscope was used for cell visualization. Cell counts were performed using the Image J software, analyzed with Prism Graphpad software, and expressed as a percentage of total $\mathrm{GFP}^{+}$cell number.

Demyelination extension. The extent of demyelination was determined based on the percentage of MOG area out of total area of the dorsal funiculus $\left(\mathrm{MOG}^{+}\right)$at $1 \mathrm{wpg}$. For each animal, 6 coronal serial sections with $120-\mu \mathrm{m}$ intervals were collected from the middle of the lesion site and quantified for different groups ( $n=3-4$ per group) of lesioned animals grafted with miPS-NPCs and mE-NPCs or injected with medium.

Cell differentiation in vivo. Grafted $\mathrm{GFP}^{+}$cells (from both cell sources) expressing Ki67, OLIG2, APC (CC1), GFAP, or NeuN were quantified on transverse sections at the lesion site to determine the proportions of proliferating cells, oligodendroglial lineage cells, astrocytes, and neurons, respectively, at different time points. For each animal, 6 coronal serial sections with $120-\mu \mathrm{m}$ intervals were collected from the middle of the lesion site. The lesion area was defined by MOG depletion, and cell density was identified by Hoechst staining of all cell nuclei on adjacent sections. Quantification was performed according to our previously published method (47). Sections were first scanned at $\times 20$ to define the limits of the lesion and level of maximal demyelination, and magnified at $\times 40$ to quantify the amount of GFP cells expressing a marker of interest. Cell counts were expressed as the percentage of total $\mathrm{GFP}^{+}$cells in the lesion area, as previously described $(2,42)$.

Cell migration in vivo. Cell dispersal within the dorsal funiculus was quantified on transverse sections evaluating the distance between the most rostral and the most caudal sections containing $\mathrm{GFP}^{+}$cells in each animal from different groups (number of sections containing $\mathrm{GFP}^{+}$cells $\times$distance between 2 sections).

Donor-derived remyelination. Exogenous myelin was visualized by MBP staining on transverse sections collected from transplanted Shi/Shi Rag2 ${ }^{-/}$(6 and $10 \mathrm{wpg}$ ). The total number of serial coronal sections containing $\mathrm{MBP}^{+}$myelin from each animal was quantified, and longitudinal distance (number of sections containing $\mathrm{MBP}^{+}$myelin $\times$ distance between 2 sections) was expressed as remyelination extension for each group. The percentage of donor-derived vs. endogenous remyelination at the lesion site was evaluated by immunostaining of the tissues with MBP (exogenous myelin) and MOG (total myelin). For each cell type, 3 levels at the Shi/Shi Rag2--spinal cord dorsal funiculus ( $1 \mathrm{~mm}$ caudal to the injection site, the injection site, and $1 \mathrm{~mm}$ rostral to the injection site; 3 serial sections with $120 \mu \mathrm{m}$ intervals per levelthus, 9 sections per animal) were quantified for $\mathrm{MBP}^{+}$or $\mathrm{MOG}^{+}$areas. Remyelination was expressed as the mean of ratios of $\mathrm{MBP}^{+}$area over $\mathrm{MOG}^{+}$area in the 3 levels in each mouse. Immunopositive areas were measured using the Image J software. Furthermore, the $\mathrm{MBP}^{+}$area was divided per number of $\mathrm{GFP}^{+}$cells on the same sections to establish the remyelination potential for miPS-NPCs versus mE-NPCs.

Furthermore, at the lesion core, the percentage of $\mathrm{NF}^{+} / \mathrm{MBP}^{+}$ axons over total number of $\mathrm{NF}^{+}$axons was quantified from 3 different fields of $1,000 \mu \mathrm{m}^{2}$ each per coronal section (on confocal images) and for 3 sections apart of $120 \mu \mathrm{m}$ intervals (a total number of 9 different fields per animal) for either miPS-NPCs or mE-NPCs grafted Shi/Shi Rag2 $2^{-/-}$mice at $6 \mathrm{wpg}$.

$G$ ratio. The $g$ ratio was determined by dividing axon circumference (for the maximum and minimum diameters of a given axon) by axon plus myelin sheath circumferences measured with the ImageJ software at a magnification of 62,000. Data were obtained from at least 50 fibers per animal and were expressed as the mean of the maximal and minimal values for each axon for adult demyelinated Shi/Shi Rag2 $2^{-/-}$mice treated with medium or grafted with different cell types.

\section{Statistics}

Data were analyzed using 2-tailed Student's $t$ test and, for multiple groups, 1-way ANOVA followed by the Tukey's multiple comparison tests. Non-normally distributed data were analyzed by the corresponding nonparametric tests. Statistical analysis was carried out using GraphPad Prism 5 software. Data were presented as mean \pm SEM for all statistical analyses. A $P$ value of less than 0.05 was considered significant.

\section{Study approval}

All mouse experiments were performed according to European Community regulations and Inserm ethical committee (authorization 75-348; 20/04/2005) and were approved by the local Darwin ethical committee. 


\section{Acknowledgments}

We acknowledge technical assistance of C. Kerninon (B. Nait Oumesmar lab, CRICM) in gene expression studies, J. Garrigue (PCR Platform, CRICM) for genotyping of animals, as well as PICPS, the Cellular Imaging Facility of the Pitié-Salpêtrière site, and the cell imaging, histology, cell culture, and rodent electrophysiology core facilities of ICM. We thank S. Goldman and M. Windrem for the transfer of Shi/Shi Rag2 $2^{-/}$mice. This work was supported by INSERM, European Leukodystrophy Foundation (ELA) ELA 2010-003C5, The US National Multiple Sclerosis Society (NMSS) RG4733A3/1, Target-
Brain EU-FP7 project (HEALTH-F2-2012-279017), NEUROKINE EU-FP7 ITN project, and the program "Investissements d'Avenir" ANR-10-IAIHU-06 and ANR-11-INBS-0011-NeurATRIS. S. Mozafari was supported by Multiple Sclerosis International Federation (www.msif.org), Ecole de Neurosciences de Paris (ENP), Région Ile de France (DIM Cerveau et Pensée), and EDF Foundation.

Address correspondence to: Anne Baron-Van Evercooren, CR-ICM, 47 bd de l'Hôpital, 75013 Paris, France. Phone: 33.1.57.27.41.23; E-mail:anne.baron@upmc.fr.
1. Martino G, Franklin RJ, Baron Van Evercooren A, Kerr DA. Stem cell transplantation in multiple sclerosis: current status and future prospects. Nat Rev Neurol. 2010;6(5):247-255.

2. Buchet D, Garcia C, Deboux C, Nait-Oumesmar B, Baron-Van Evercooren A. Human neural progenitors from different foetal forebrain regions remyelinate the adult mouse spinal cord. Brain. 2011;134(pt 4):1168-1183.

3. Uchida N, et al. Human neural stem cells induce functional myelination in mice with severe dysmyelination. Sci Transl Med. 2012;4(155):155ra36.

4. Windrem MS, et al. Neonatal chimerization with human glial progenitor cells can both remyelinate and rescue the otherwise lethally hypomyelinated shiverer mouse. Cell Stem Cell. 2008;2(6):553-565.

5. Windrem MS, et al. A competitive advantage by neonatally engrafted human glial progenitors yields mice whose brains are chimeric for human glia. J Neurosci. 2014;34(48):16153-16161.

6. Czepiel M, et al. Differentiation of induced pluripotent stem cells into functional oligodendrocytes. Glia. 2011;59(6):882-892.

7. Pouya A, Satarian L, Kiani S, Javan M, Baharvand $H$. Human induced pluripotent stem cells differentiation into oligodendrocyte progenitors and transplantation in a rat model of optic chiasm demyelination. PLoS One. 2011;6(11):e27925.

8. Wang S, et al. Human iPSC-derived oligodendrocyte progenitor cells can myelinate and rescue a mouse model of congenital hypomyelination. Cell Stem Cell. 2013;12(2):252-264.

9. Kim J, et al. Direct reprogramming of mouse fibroblasts to neural progenitors. Proc Natl Acad Sci U S A. 2011;108(19):7838-7843.

10. Kim SM, et al. Direct conversion of mouse fibroblasts into induced neural stem cells. Nat Protoc. 2014;9(4):871-881.

11. Ring KL, et al. Direct reprogramming of mouse and human fibroblasts into multipotent neural stem cells with a single factor. Cell Stem Cell. 2012;11(1):100-109.

12. Zou Q, et al. Direct conversion of human fibroblasts into neuronal restricted progenitors. J Biol Chem. 2014;289(8):5250-5260.

13. Thier M, et al. Direct conversion of fibroblasts into stably expandable neural stem cells. Cell Stem Cell. 2012;10(4):473-479.

14. Najm FJ, et al. Transcription factor-mediated reprogramming of fibroblasts to expandable, myelinogenic oligodendrocyte progenitor cells. Nat Biotechnol. 2013;31(5):426-433.
15. Yang N, et al. Generation of oligodendroglial cells by direct lineage conversion. Nat Biotechnol. 2013;31(5):434-439.

16. Keirstead HS, Ben-Hur T, Rogister B, O'Leary MT, Dubois-Dalcq M, Blakemore WF. Polysialylated neural cell adhesion molecule-positive CNS precursors generate both oligodendrocytes and Schwann cells to remyelinate the CNS after transplantation. J Neurosci. 1999;19(17):7529-7536.

17. Vitry S, Avellana-Adalid V, Lachapelle F, BaronVan Evercooren A. Migration and multipotentiality of PSA-NCAM+ neural precursors transplanted in the developing brain. Mol Cell Neurosci. 2001;17(6):983-1000.

18. Laterza C, et al. iPSC-derived neural precursors exert a neuroprotective role in immune-mediated demyelination via the secretion of LIF. Nat Commun. 2013;4:2597.

19. Eftekharpour E, Karimi-Abdolrezaee S, Wang J, El Beheiry H, Morshead C, Fehlings MG. Myelination of congenitally dysmyelinated spinal cord axons by adult neural precursor cells results in formation of nodes of Ranvier and improved axonal conduction. J Neurosci. 2007;27(13):3416-3428.

20. Love S. Demyelinating diseases. J Clin Pathol. 2006;59(11):1151-1159.

21. Han DW, et al. Direct reprogramming of fibroblasts into neural stem cells by defined factors. Cell Stem Cell. 2012;10(4):465-472.

22. Lujan E, Chanda S, Ahlenius H, Sudhof TC, Wernig M. Direct conversion of mouse fibroblasts to self-renewing, tripotent neural precursor cells. Proc Natl Acad Sci US A. 2012;109(7):2527-2532.

23. Matsui T, et al. Neural stem cells directly differentiated from partially reprogrammed fibroblasts rapidly acquire gliogenic competency. Stem Cells. 2012;30(6):1109-1119.

24 . Niu W, et al. In vivo reprogramming of astrocytes to neuroblasts in the adult brain. Nat Cell Biol. 2013;15(10):1164-1175.

25. Zappone MV, et al. Sox2 regulatory sequences direct expression of a (beta)-geo transgene to telencephalic neural stem cells and precursors of the mouse embryo, revealing regionalization of gene expression in CNS stem cells. Development. 2000;127(11):2367-2382.

26. Czepiel M, Leicher L, Becker K, Boddeke E, Copray S. Overexpression of polysialylated neural cell adhesion molecule improves the migration capacity of induced pluripotent stem cell-derived oligodendrocyte precursors. Stem
Cells Transl Med. 2014;3(9):1100-1109.

27. Tsuji O, et al. Therapeutic potential of appropriately evaluated safe-induced pluripotent stem cells for spinal cord injury. Proc Natl Acad Sci U S A. 2010;107(28):12704-12709.

28. Okita K, Nakagawa M, Hyenjong H, Ichisaka T, Yamanaka S. Generation of mouse induced pluripotent stem cells without viral vectors. Science. 2008;322(5903):949-953.

29. Nakagawa M, et al. Generation of induced pluripotent stem cells without Myc from mouse and human fibroblasts. Nat Biotechnol. 2008;26(1):101-106.

30. Nash B, Ioannidou K, Barnett SC. Astrocyte phenotypes and their relationship to myelination. JAnat. 2011;219(1):44-52.

31. Crawford AH, Chambers C, Franklin RJ. Remyelination: the true regeneration of the central nervous system. JComp Pathol. 2013;149(2-3):242-254.

32. Zujovic V, Bachelin C, Baron-Van Evercooren A. Remyelination of the central nervous system: a valuable contribution from the periphery. Neuroscientist. 2007;13(4):383-391.

33. Gout O, Gansmuller A, Baumann N, Gumpel M. Remyelination by transplanted oligodendrocytes of a demyelinated lesion in the spinal cord of the adult shiverer mouse. Neurosci Lett. 1988;87(1-2):195-199.

34. Chomiak T, Hu B. What is the optimal value of the g-ratio for myelinated fibers in the rat CNS? A theoretical approach. PLoS One. 2009;4(11):e7754.

35. Brinkmann BG, et al. Neuregulin-1/ErbB signaling serves distinct functions in myelination of the peripheral and central nervous system. Neuron. 2008;59(4):581-595.

36. Mozafari S, Sherafat MA, Javan M, Mirnajafi-Zadeh J, Tiraihi T. Visual evoked potentials and MBP gene expression imply endogenous myelin repair in adult rat optic nerve and chiasm following local lysolecithin induced demyelination. Brain Res. 2010;1351:50-56.

37. Smith KJ, Bostock H, Hall SM. Saltatory conduction precedes remyelination in axons demyelinated with lysophosphatidyl choline. J Neurol Sci. 1982;54(1):13-31.

38. Smith KJ, McDonald WI, Blakemore WF. Restoration of secure conduction by central demyelination. Trans Am Neurol Assoc. 1979;104:25-29.

39. Utzschneider DA, Archer DR, Kocsis JD, Waxman SG, Duncan ID. Transplantation of glial cells enhances action potential conduction of amyelinated spinal cord axons in the myelin-deficient rat. Proc Natl Acad Sci US A. 
1994;91(1):53-57.

40. Blakemore WF, Gilson JM, Crang AJ. Transplanted glial cells migrate over a greater distance and remyelinate demyelinated lesions more rapidly than endogenous remyelinating cells. J Neurosci Res. 2000;61(3):288-294.

41. Deboux C, et al. Overexpression of CD44 in neural precursor cells improves trans-endothelial migration and facilitates their invasion of perivascular tissues in vivo. PLoS One. 2013;8(2):e57430.

42. Blanchard B, et al. Tocopherol derivative TFA-12 promotes myelin repair in experimen- tal models of multiple sclerosis. J Neurosci. 2013;33(28):11633-11642.

43. Shinkai Y, et al. RAG-2-deficient mice lack mature lymphocytes owing to inability to initiate V(D)J rearrangement. Cell. 1992;68(5):855-867.

44. Eftekharpour E, Karimi-Abdolrezaee S, Sinha K, Velumian AA, Kwiecien JM, Fehlings MG. Structural and functional alterations of spinal cord axons in adult Long Evans Shaker (LES) dysmyelinated rats. Exp Neurol. 2005;193(2):334-349.

45. Iuliano BA, Schmelzer JD, Thiemann RL, Low PA, Rodriguez M. Motor and somatosensory evoked potentials in mice infected with Theiler's murine encephalomyelitis virus. J Neurol Sci. 1994;123(1-2):186-194.

46. Walczak P, et al. Human glial-restricted progenitors survive, proliferate, and preserve electrophysiological function in rats with focal inflammatory spinal cord demyelination. Glia. 2011;59(3):499-510.

47. Girard C, et al. Grafts of brain-derived neurotrophic factor and neurotrophin 3-transduced primate Schwann cells lead to functional recovery of the demyelinated mouse spinal cord. JNeurosci. 2005;25(35):7924-7933. 